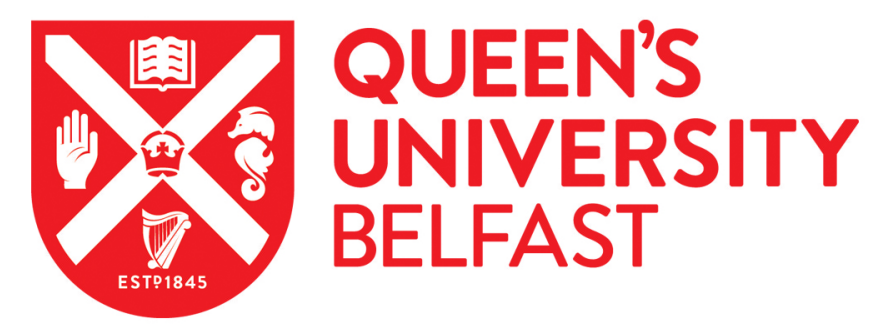

\title{
Global metabolite profiles of rice brown planthopper-resistant traits reveal potential secondary metabolites for both constitutive and inducible defenses
}

Uawisetwathana, U., Chevallier, O. P., Xu, Y., Kamolsukyeunyong, W., Nookaew, I., Somboon, T., Toojinda, T., Vanavichit, A., Goodacre, R., Elliott, C. T., \& Karoonuthaisiri, N. (2019). Global metabolite profiles of rice brown planthopper-resistant traits reveal potential secondary metabolites for both constitutive and inducible defenses. Metabolomics, 15(12), [151]. https://doi.org/10.1007/s11306-019-1616-0

Published in:

Metabolomics

Document Version:

Peer reviewed version

Queen's University Belfast - Research Portal:

Link to publication record in Queen's University Belfast Research Portal

Publisher rights

() 2019 Springer Science+Business Media, LLC, part of Springer Nature.

This work is made available online in accordance with the publisher's policies. Please refer to any applicable terms of use of the publisher.

\section{General rights}

Copyright for the publications made accessible via the Queen's University Belfast Research Portal is retained by the author(s) and / or other copyright owners and it is a condition of accessing these publications that users recognise and abide by the legal requirements associated with these rights.

Take down policy

The Research Portal is Queen's institutional repository that provides access to Queen's research output. Every effort has been made to ensure that content in the Research Portal does not infringe any person's rights, or applicable UK laws. If you discover content in the

Research Portal that you believe breaches copyright or violates any law, please contact openaccess@qub.ac.uk. 


\section{A list of response to the comments}

Reviewer \#2: The revised manuscript entitled "Global metabolite profiles of rice brown planthopper-resistant traits reveal potential secondary metabolites for both constitutive and inducible defenses" is suitable for publication in Metabolomics after correction of a few details. Most remarks made by the two reviewers have been taken into account and addressed properly.

\section{Introduction}

L116: Do you mean "metabolites involved in taurine and hypotaurine metabolism » or "taurine and hypotaurine".

Response: We have removed the word "metabolism" as shown in Line 115, pg. 6.

\section{Methods}

Part of the response to the remark about the extraction protocol (initially L 165) could be rephrased and added in the Discussion section as a sentence with one or two references.

Response: We have added the sentences according to your suggestion in Discussion section (Line 480-484, pg. 21).

"This study found flavonoid glycoside (MSI level 1), chlorogenic acid (MSI level 1) and hydroxytriterpenoid (MSI level 2) in the resistant rice variety. With hydrophilic as their functional groups, these compounds are water soluble as reported in these studies (Matsui et al., 2004, Slámová et al., 2018) which could be extracted by methanol/water used in this study"

Typo error L283: were manually matched to their monoisotopic masses Response: We have added the word "to" as shown in Line 276, pg. 12.

Typo error L262: were tested for significant differences

Response: We have added the word "for" as shown in in Line 256, pg. 12.

L268: MTBLS1197 is not listed on MetaboLights website yet. Check that it will be soon. Response: We have checked into the system and there were some technical problem on the author's side to be corrected. Currently, MTBLS1 197 is now validated and will be listed on MetaboLights soon.

\section{Results}

L348: "metabolite features with FDR $<0.001$ and p-value $<0.05$ " is ambiguous. Do you mean "metabolite features with adjusted p-value $<0.05$ after FDR correction" or similar?

Response: We have revised as suggested in Line 335, pg. 15.

Typo error L396-397: The first subgroup, constitutive response (Cluster IIIa of ESI-: 9 features), represented metabolites whose levels were significantly higher ...

Response: We have made changed as suggested in Line 383, pg. 17.

Typo error L438: those in the Il308 were not changed

Response: We have revised the word according to your suggestion in Line 422, pg. 18. 
L445: "but" does not seem necessary.

Response: We have made changed as suggested in Line 429, pg. 19.

L453-454: acetate metabolite levels were constitutively higher (4.7 and 2.5 fold increase, respectively) than those in...

Response: We have moved the word according to your suggestion in Line 435, pg. 19.

\section{Discussion}

Typo error L 483: These results

Response: We have made changed as suggested in Line 448, pg. 20.

L424-425: Flavonoid glycosides are diverse across plant species.

Response: We have made changed according to your suggestion in Line 489, pg. 21.

\section{Figures}

Figure 1 legend: mention MS in the legend.

Response: We have mentioned the MS in the legend as shown in Line 595, pg. 25. 


\section{Global metabolite profiles of rice brown planthopper-resistant traits reveal}

2 potential secondary metabolites for both constitutive and inducible

3 defenses

4

5 Umaporn Uawisetwathana $^{\mathrm{a} *}$, Olivier P. Chevallier ${ }^{\mathrm{b}},{\mathrm{Yun} \mathrm{Xu}^{\mathrm{c}} \text {, Wintai Kamolsukyeunyong }}^{\mathrm{d}}$,

6 Intawat Nookaew $^{\mathrm{e}}$, Thapakorn Somboon ${ }^{\mathrm{a}}$, Theerayut Toojinda ${ }^{\mathrm{d}, \mathrm{f}}$, Apichart Vanavichit ${ }^{\mathrm{g}}$,

$7 \quad$ Royston Goodacre ${ }^{\mathrm{c}}$, Christopher T. Elliott ${ }^{\mathrm{b}}$, Nitsara Karoonuthaisiri ${ }^{\mathrm{a}}$

8

$9 \quad{ }^{a}$ Microarray Laboratory, National Center for Genetic Engineering and Biotechnology

10 (BIOTEC), National Science and Technology Development Agency (NSTDA), Khlong

11 Nueng, Khlong Luang, Pathumthani 12120, Thailand

$12{ }^{b}$ Institute for Global Food Security, School of Biological Sciences, Queen's University

13 Belfast, Stranmillis Road, Belfast BT9 5AG, Northern Ireland

$14{ }^{\mathrm{c}}$ Department of Biochemistry, Institute of Integrative Biology, University of Liverpool,

15 Liverpool L69 3BX, United Kingdom

$16{ }^{\mathrm{d}}$ Rice Gene Discovery and Utilization Laboratory, Innovative Plant Biotechnology and

17 Precision Agriculture Research Team, National Center for Genetic Engineering and

18 Biotechnology (BIOTEC), National Science and Technology Development Agency

19 (NSTDA), Khlong Luang, Pathum Thani, Thailand

$20{ }^{\mathrm{e}}$ College of Medicine, Department Biomedical Informatics, University of Arkansas for

21 Medical Sciences, Little Rock, AR, United States

$22{ }^{\mathrm{f}}$ Integrative Crop Biotechnology and Management Research Group, National Center for

23 Genetic Engineering and Biotechnology (BIOTEC), National Science and Technology

24 Development Agency (NSTDA), Khlong Luang, Pathum Thani, Thailand. 
$25{ }^{\mathrm{g}}$ Agronomy Department, Faculty of Agriculture at Kamphaeng Saen, Kasetsart University, 26 Kamphaeng Saen, Nakhon Pathom, Thailand.

27

$28 *$ corresponding author

29 Tel.: +66-2-5646700 ext $3265 \quad$ Fax: +66-2-5646707

30 Email: umaporn.uaw@biotec.or.th

31 Abbreviated title: Rice metabolomics analysis of brown planthopper-resistant traits 


\section{Abstract}

Brown planthopper $(\mathrm{BPH})$ is a phloem feeding insect that causes annual disease outbreaks, called hopper burn in many countries throughout Asia, resulting in severe damage to rice production. Currently, mechanistic understanding of $\mathrm{BPH}$ resistance in rice plant is limited, which has caused slow progression on developing effective rice varieties as well as effective farming practices against $\mathrm{BPH}$ infestation. To reveal rice metabolic responses during 8 days of $\mathrm{BPH}$ attack, this study examined polar metabolome extracts of $\mathrm{BPH}$ susceptible (KD) and its BPH-resistant isogenic line (IL308) rice leaves using UPLC-QToFMS combined with multi-block PCA. This multivariate statistical model revealed different metabolic response patterns between the $\mathrm{BPH}$-susceptible and $\mathrm{BPH}$-resistant varieties during BPH infestation. The metabolite responses of the resistant IL308 variety occurred on Day 1, which was significantly earlier than those of the susceptible KD variety which showed an induced response by Days 4 and 8 . BPH infestation caused metabolic perturbations in purine, phenylpropanoid, flavonoid, and terpenoid pathways. While found in both susceptible and resistant rice varieties, schaftoside (1.8 fold), iso-schaftoside (1.7 fold), rhoifolin (3.4 fold) and apigenin6-C- $\alpha$-L-arabinoside- $8-C-\beta$-L-arabinoside levels (1.6 fold) were significantly increased in the resistant variety by Day 1 post-infestation. 20-hydroxyecdysone acetate $(2.5$ fold) and dicaffeoylquinic acid (4.7 fold) levels were considerably higher in the resistant rice variety than those in the susceptible variety, both before and after infestation, suggesting that these secondary metabolites play important roles in inducible and constitutive defenses against the BPH infestation. These potential secondary metabolites will be useful as metabolite markers and/or bioactive compounds for effective and durable approaches to address the BPH problem.

Keywords: LC-HRMS; multi-block principal component analysis; Metabolite profiling; Brown planthopper resistance; Thai Jasmine rice; Oryza sativa 


\section{Introduction}

Rice is the staple food of more than half of the world's population. Thai Jasmine rice is recognized worldwide for its fine quality due to its jasmine scent and unique texture

(Wongpornchai et al., 2002). However, this rice variety is highly susceptible to insect infestation, especially brown planthopper (BPH), which causes devastating production yield losses (Heong, 2009). Pesticides have been used to control the disease, but they are toxic and harmful to humans and their residues can contaminate the crops (Heinrichs, 1979). To

overcome this problem with an environmentally-friendly and sustainable production strategy, rice breeding programs have successfully produced $\mathrm{BPH}$-resistant isogenic lines of Thai Jasmine rice using a backcross introgression technique from the donor BPH-resistant Rathu Heenati (RH) to the recipient Thai Jasmine rice (Toojinda et al., 2005, Jairin et al., 2009).

The resulting rice isogenic lines conserved the aroma and texture characteristics of Thai Jasmine rice, as well as carrying BPH resistance loci from RH rice variety.

The $\mathrm{RH}$ rice variety, a native rice from Sri Lanka, is well known as possessing a broad spectrum range of BPH resistance to four of the most dominant BPH biotypes (Fujita et al., 2013). In the post-genomic era, linkage map analysis showed that RH variety contains three main BPH resistance loci on chromosomes 4, 6 and 10 (Hu et al., 2016). Two major BPH resistant genes (Bph3 and Bph32) have been characterized by map-based cloning. Bph3 located on short arm of chromosome 4 was identified as a plasma-membrane-localized lectin receptor kinase (Liu et al., 2015), and Bph32 located on short arm of chromosome 6 was identified as an unknown short consensus repeat (SCR) domain-containing protein localized on the plasma membrane of the cells. The BPH-resistant rice varieties conferred an antibiosis resistance to BPH and Bph32 is highly expressed in the leaf sheaths (Ren et al., 2016). The introgression line carrying Bph3 and Bph32 showed an increased expression level of the OsSTPS2 (sesquiterpene synthase 2) transcript located on chromosome 4 
82 (Kamolsukyeunyong et al., 2019). Pitija et al. also reported elevated levels of monoterpenoids 83 in the BPH-resistant rice varieties (IL162, IL283, IL302 and IL308) when compared to the susceptible KD cultivar using GC-MS analysis (Pitija et al., 2014). Moreover, LOC_Os06g03514 transcript (a putative metallopeptidase), a gene involved in catalyzing reactive oxygen species, was induced in the resistant rice variety after BPH infestation (Kusumawati et al., 2018). Feeding and behavioral studies revealed that BPH preferred settling on susceptible Taichung Native 1 (TN1) to BPH-resistant IL308 variety (Kamolsukyunyong et al., 2013) and did not prefer feeding on BPH-resistant RH variety as determined by lower honeydew excretion compared to the susceptible variety (Kusumawati et al., 2018), suggesting that the BPH-resistant rice showed antixenosis and antibiosis activities. The elucidation of BPH resistance loci from the previous studies were conducted at gene structure and gene expression level. Recently, high-resolution mapping and breeding technique has been applied to identify a novel brown planthopper resistance gene derived from wild rice (Oryza. rufipogon Griff) (Li et al., 2019). However, there is still a lack of knowledge regarding the metabolic response mechanism associated with BPH infestation between susceptible and resistant rice (carrying Bph3 and Bph32) lines that need further investigation to provide better understanding of BPH resistance mechanisms.

Plants generally respond to abiotic and biotic stresses by producing different metabolites, particularly, secondary metabolites which play significant roles in plant defense mechanism against herbivores attack (Balmer et al., 2013, Nakabayashi and Saito, 2015). Previous studies employed ${ }^{1} \mathrm{H}$ NMR-based metabolomics using methanol-water extraction to identify primary metabolic response of BPH-resistant rice carrying Bph 14 (Liu et al., 2010, Liu et al., 2017) and Bph3 (Uawisetwathana et al., 2015) and revealed that metabolites involved in GABA shunt, glycolysis, amino acid biosynthesis, purine/pyrimidine biosynthesis changed in response to BPH infestation. Gas chromatography-based metabolomics using 
methanol-hexane extraction was employed to study primary and secondary metabolic responses of rice carrying Bph6 revealing sterol and phytol biosyntheses as important metabolic pathways against BPH attack (Zhang et al., 2018). In addition, a combination of liquid chromatography-based metabolomics using methanol-water extraction and gas chromatography-based metabolomics using methoxy-amino-hydrochloride and MSTFA derivatization was used to reveal changes in levels of several metabolites such as cyanoamino acids, thiamine, taurine and hypotaurine, and quercetin and spermidine metabolites as a response in rice carrying Bph1, bph2, Bph3 and Bph32 under BPH infestation (Kang et al., 2019). These studies suggested that metabolites contributing to particular BPH resistant genes in rice were diverse. However, metabolites, especially secondary metabolites related to the rice carrying only $B p h 3$ and $B p h 32$, have not been explicitly identified yet.

To investigate plant metabolites under biotic stress condition, ultra-high performance liquid chromatography-quadrupole time of flight-mass spectrometry (UHPLC-QToF-MS) was employed as it is a powerful tool with high resolution which provides excellent mass accuracy, as well as its wide metabolites coverage which supports the detection of both primary and secondary metabolites (Allwood and Goodacre, 2010). In combination with multivariate statistical analysis, multi-block principal component analysis (MBPCA) has been shown to accelerate data fusion and data interpretation, particularly in the experiments influenced by multiple factors (Xu and Goodacre, 2012). Successful application of MS-based metabolomics in combination with MBPCA has been used for metabolite profiling such as to study multi-platforms metabolome in melon (Biais et al., 2009), to monitor the natural spoilage process in pork (Xu et al., 2013) and to study the effects of post flowering environmental conditions on blackcurrant fruit (Allwood et al., 2019).

In this study, UPLC-QToF-MS was employed to profile and pinpoint the key metabolites differentially altered between the BPH-susceptible Thai Jasmine rice and its 
132

BPH-resistant isogenic line (IL308). The comparison of the samples with and without BPH infestation for three different time points (Days 1, 4 and 8) revealed that a specific group of secondary metabolites involved in phenylpropanoid, flavonoid or terpenoid metabolism plays a significant role in both constitutive and inducible defenses in the rice samples.

\section{Materials and Methods}

\section{Rice and brown planthopper (BPH) samples}

Brown planthopper (BPH, Nilaparvata lugens) biotype Ubon Rachathani (UBN-BPH) was cultured in a standard BPH-susceptible Taichung Native 1 (TN1) rice cultivar in a closed system. Paddy soil without fertilizer was used for planting of TN1 in the pots.

Approximately, 50-100 TN1 seeds were germinated on each pot. Two to three-week-old seedlings of TN1 were used for insect cultivation. The reared $\mathrm{BPH}$ population at third to fourth-instar nymph stage was used for rice infestations (Jairin et al., 2009).

Rice samples used in this study were BPH-susceptible Thai jasmine rice KDML105

(KD) and BPH-resistant isogenic line (UBN3078-101-432-6-308: IL308). IL308 is the BPHresistant IL of KD containing Bph3, Bph 32 and OsSTPS2 (Liu et al., 2015, Ren et al., 2016, Kamolsukyeunyong et al., 2019).

\section{BPH treatment and rice sample collection}

The BPH treatment was performed as depicted in Figure S1. Paddy soil collected from the field was used in this experiment. Rice seeds were germinated in the pots for one week. Then, water was added into each pot to cover the soil. At 3-week-old seedling stage, one gram of nitrogen fertilizer (46-0-0) was added to each pot. The water was always maintained to cover the soil during the experiment. All rice varieties examined in this study were grown in a total of 36 pots (18 pots/each variety; pot dimensions: $20 \mathrm{~cm}$ in diameter and $18 \mathrm{~cm}$ in height) with 3 plants per pot in a greenhouse with $13 \mathrm{~h}(06: 00-19: 00)$ light and $11 \mathrm{~h}$ 
(19:00 - 06:00) dark cycles for 45 days (tilling stage) before dividing the rice samples into two groups (with and without BPH treatment, 9 pots/group). For the BPH treatment group, each rice variety was transferred into an aluminum cage covered with plastic mesh $(1 \mathrm{~mm}$ diameter) and second- to third-instar BPH nymphs were then introduced to each plant (100$150 \mathrm{BPH}$ nymphs/pot). In parallel, the groups without BPH treatment were maintained in separate cages as control groups.

Three biological replicates (three separate pots) of the rice leaf samples in each variety were collected at three different time points (Days 1, 4, and 8). The samples including leaf sheathes and leaf blades were cut on the lowest point of the leaf sheath (approximately 60-100 cm tall) before quickly freezing in liquid nitrogen. All frozen samples were lyophilized (Modulyo®, Thermo Scientific, USA), ground to a fine powder (Phillip HR2020, USA) and stored at $-80^{\circ} \mathrm{C}$ prior to analysis.

\section{Metabolite extraction}

An in-house extraction protocol was used in this study with some modifications 171 (Carrizo et al., 2017, O'Kane et al., 2013). Freeze-dried leaf powder (100 mg) was extracted

with $2 \mathrm{~mL}$ of 3:2(v/v) methanol:water solution by mixing for $10 \mathrm{~min}$ at room temperature using a minimix standard shaker (Merris Enginering, U.K.) before sonicating for $15 \mathrm{~min}$ at $4^{\circ} \mathrm{C}$ in a ultrasonic bath sonicator. Extracts were centrifuged at $3,500 \times g$ at $4^{\circ} \mathrm{C}$ for $20 \mathrm{~min}$ (Sorvall Legend, Thermo Scientific). Supernatant was collected for polar metabolome analysis. Supernatants $(1 \mathrm{~mL}$ each) were dried in a vacuum concentrator (MiVac, Genevac, $\mathrm{UK})$ at $35^{\circ} \mathrm{C}$ for $24 \mathrm{~h}$ before being reconstituted in $200 \mu \mathrm{L}$ Ultra-Pure water and filtered using $0.22 \mu \mathrm{m}$ Costar Spin-X® Centrifuge Tube Filter $\left(10,000 \times g\right.$ at $4^{\circ} \mathrm{C}$ for $\left.5 \mathrm{~min}\right)$. The flow-through $(150 \mu \mathrm{L})$ was collected and transferred to a HPLC glass vial with a micro insert prior to ultra-high pressure liquid chromatography-quadrupole time of flight-mass spectrometry (UPLC-QToF-MS) analysis. 


\section{Untargeted metabolite profiles using LC-QToF-MS}

Filtered extracts were analysed using an Acquity UPLC I-class system (Waters, MA, USA) which coupled an Acquity UPLC® HSS T3 $(2.1$ x $100 \mathrm{~mm}, 1.8 \mu \mathrm{m})$ column (Waters, USA) with a Xevo G2S QToF mass spectrometer (Waters, MA, USA). The flow rate was 0.4 $\mathrm{mL} / \mathrm{min}$ and the column temperature was maintained at $45^{\circ} \mathrm{C}$. Mobile phases were water with $0.1 \%$ formic acid (A) and methanol with $0.1 \%$ formic acid (B). Sample manager temperature was set at $6^{\circ} \mathrm{C}$ and an aliquot of $5 \mu \mathrm{L}$ was injected onto the column using the following method: an isocratic period for 0.50 min at 99:1 (A:B) followed by a linear gradient to 1:99 (A:B) over 16.50 min which was maintained for $1.75 \mathrm{~min}$ before restoring to an initial condition (99:1, A:B) for 1.25 min. For reproducibility measurement of the instrument, pooled biological samples were used as quality control samples injected every 10 samples (Dunn et al., 2011, Broadhurst et al., 2018). Prior to the experiment, system suitability (LC and MS) was assessed by 3 consecutive injections of commercially available reference standard (Waters "test mix", part 186006963). A lock-mass solution of Leucine Enkephalin $(1 \mathrm{ng} / \mu \mathrm{L})$ in acetonitrile/water containing $0.1 \%$ formic acid $(1: 1, \mathrm{v} / \mathrm{v})$ was continuously infused into the MS via the lock-spray at a flow rate of $10 \mu \mathrm{L} / \mathrm{min}$. Each filtered extract was randomly injected three times to obtain three technical replicates throughout the experiment $(n=3 \times 3)$. From the nine values, the mean was calculated. The means from all groups were compared and statistically analyzed as described in details in data fusion and data analysis section.

The MS data were acquired in both positive and negative electrospray ionization (ESI) modes over the range 50-1200 Da. The ionization source parameters in positive mode were as follows: capillary voltage $1.0 \mathrm{kV}$, sample cone voltage $30 \mathrm{~V}$, source temperature $120^{\circ} \mathrm{C}$, desolvation temperature $450^{\circ} \mathrm{C}$, desolvation gas flow $850 \mathrm{~L} / \mathrm{h}$ and cone gas flow 25 $\mathrm{L} / \mathrm{h}$. For negative mode, the following settings were applied: capillary voltage $2.0 \mathrm{kV}$, sample 
cone $30 \mathrm{~V}$, source temperature $120^{\circ} \mathrm{C}$, desolvation temperature $450^{\circ} \mathrm{C}$, desolvation gas flow $850 \mathrm{~L} / \mathrm{h}$ and cone gas flow $25 \mathrm{~L} / \mathrm{h}$. Mass spectra data were acquired in continuum mode with a scan time of $0.1 \mathrm{sec}$ using MSE function (low energy: $4 \mathrm{eV}$, high energy: ramp from 10 to $30 \mathrm{eV}$ in both positive and negative modes).

Spectra from the LC-MS analysis were analysed using MassLynx version 4.1 and Progenesis QI softwares (Waters, USA). Briefly, raw chromatogram data acquired by MassLynx software were transformed into 2D matrices between retention time and $\mathrm{m} / \mathrm{z}$. The data were preprocessed using Progenesis QI software by alignment and peak picking. For peak picking, a minimum peak width filter of 0.06 min was applied. Progenesis QI uses ratiometric data in log space, along with a median and mean absolute deviation outlier filtering approach, to calculate the scalar factor for normalization and is able to determine which pairs (or groups) of compound ions might be different adduct forms of the same compound. For 2 compound ions to be considered part of the same compound they must have the same retention time and ion masses that differ by an amount equal to the mass difference between 2 experiments. These programs quantify and aid identification of the integrals from all peaks above the threshold deemed to be of interest.

\section{Data fusion and data analyses}

Principal component analysis was applied to auto-scaled data matrices for first visualization and assess the stability of the analytical platform. Multi-block principal component analysis (MBPCA) was then employed for two purposes: (1) to fuse both positive and negative mode data together, and (2) visualize the pattern of the data as a whole (Xu et al., 2013). The data collected from both LC-MS positive and negative modes were placed side-by-side and matched on the sample level (i.e. each row is the same sample analysed on positive and negative mode respectively). Each block was firstly auto-scaled so that the mean and standard deviation of each variable were zero and 1, respectively. Block scaling (i.e. the 
data within each block were divided by the square root of the number of variables in that block) was then applied to compensate for the variance differences caused by different number of variables in different modes. Consensus principal component analysis (CPCA), a commonly used MBPCA model (Smilde et al., 2003), was then applied to gain a consensus view of the pattern of the data collected from both ionization modes.

Another type of analysis is to decouple the effects from multiple influential factors (Xu and Goodacre, 2012). In this study, there are three main factors: temporal factor, genotypes, and treatment of BPH. To be able to view the effect of one factor without interference from the other factors, CPCA was applied to both ESI positive and negative ion modes separately. On each mode of data, the samples were partitioned to three blocks according to time, each block has all the samples on the same time point while between different blocks the samples were matched on both genotype and treatment; i.e., on each row the samples on the three blocks have the same genotype and BPH treatment (either treated or control). Auto-scaling was applied to each data block prior to CPCA.

Screening workflow was shown in Figure 1. In the first screening, three-way analysis of variance (3-way ANOVA) with false discovery rate $<0.001$ and $p$-value $<0.05$ has been employed to identify metabolites which varied significantly at least genotype factor, interactions by genotype/treatment, genotype/time and genotype/treatment/time. In the second screening, the selected metabolite features were transformed to log value and auto scaled prior to hierarchical clustering based on metabolite patterns with their level changes $>1.5$ folds using Cluster 3.0 software (Eisen et al., 1998). In the third screening, the selected metabolite features were tested for significant differences between control and treatment groups per time per genotype using Mann-Whitney U test $(* p<0.05$, $* * p<0.01$ and $* * *$ $p<0.001)$. The response of each sample ( $\mathrm{n}=3 \times 3$ /group) was calculated as the mean values with standard deviation (SD). Multi-block analysis and three-way ANOVA were performed 
in MATLAB 2016a (Mathworks, MA, U.S.A). Mann-Whitney U test was performed in

258 GraphPad Prism 5.01 (GraphPad Software, San Diego, CA). The metadata was available at 259 the MetaboLights metabolomics data repository with an identifier MTBLS1197 (URL

260 www.ebi.ac.uk/metabolights/MTBLS1197).

\section{Results}

\section{Metabolite identification}

Metabolite features were identified according to the minimum reporting standards for chemical analysis defined by the Metabolomics Standards Initiative (MSI) (Sumner et al., 2007). The ions with identified adduct forms and neutral masses were automatically matched to their MS spectra and monoisotopic masses using external public databases including ChemSpider (http://www.chemspider.com/), HMDB (Wishart et al., 2013), BioCyc (http://biocyc.org/), Plant Metabolic Network (http://www.plantcyc.org/), NIST (http://www.nist.gov/), LipidMAPS (http://www.lipidmaps.org/) and KEGG (http://www.genome.jp/kegg/) provided in Progenesis software. The parameter criteria were composed of precursor tolerance at $10 \mathrm{ppm}$, fragment tolerance at $10 \mathrm{ppm}$ and isotope similarity filter at $95 \%$. Additionally, the selected ions that could not be identified from aforementioned databases were manually matched to their monoisotopic masses using Metlin (https://metlin.scripps.edu). Precursor tolerance was set at $30 \mathrm{ppm}$. For MSI level 1, the identities of metabolite features were confirmed by using authentic standards (isoschaftoside, schaftoside, dicaffeoylquinic acid; Sigma, USA). For MSI level > 1, the identities of these metabolite features were annotated based on MS spectra (precursor ions and their pseudo MS/MS acquired by $\mathrm{MS}^{\mathrm{E}}$ function of QToF-MS) using the public databases above. 


\section{Phenotypic response affected by BPH infestation}

The temporal effect of BPH infestation on rice leaf between susceptible and resistant rice varieties was investigated. First, leaves from both rice varieties were visibly the same without BPH infestation, while those in the BPH-treated groups were clearly different on Day

4 and more obvious by Day 8 (Figure 2). Specifically, the leaf samples in the BPH-

susceptible KD cultivar (KD) turned yellow and dried, exhibiting the classical hopper burn symptoms, whereas those of the BPH-resistant IL308 variety (IL308) were not affected by the infestation. Liquid chromatography-high resolution mass spectrometry (UPLC-QToF-

MS) was then employed to determine metabolic response associated with the BPH infestation.

\section{Global metabolic response affected by BPH infestation}

Base peak intensity (BPI) chromatograms of the samples in this study showed

differences across the rice varieties, along the time points and between them with and without $\mathrm{BPH}$ treatment in both positive electrospray ionization (ESI+) and negative electrospray ionization (ESI-) modes. Representative BPI chromatograms of BPIs of the leaf extracts are shown in Figure S2.

Following peak peaking, the data were normalized within the Progenesis software (4,688 ions for ESI+ mode and 2,151 ions for ESI- mode) as detailed in the Materials and Methods, and were then subjected to PCA and MBPCA for visualization of global metabolic profiles among the sample groups. The scores plot of PCA was given in Figure $\mathbf{S 3}$ and it showed that all the QC samples were tightly clustered together, indicating that the analytical platform was stable during the course of analysis. Unlike classical PCA, MBPCA models such as CPCA produce two sets of scores, namely super scores and block scores. The super scores showed "common trend" across different blocks, while block scores showed unique patterns on each block. The MBPCA super scores plot revealed distinctive differences among 
the time points of the experiment, and showed slight differences on the BPH treatment with 44.4\% total explained variance (TEV) in PC1 and 13.5\% TEV in PC2. However, these analyses did not show obvious differences between the rice varieties (Figure S4). This result suggested that the temporal effect of the experiment was a major influential factor for the separation between the metabolic profiles which corresponding to the occurrence of the hopper burn. Consequently, further time block scores plots were performed to remove the temporal factor in the dataset in order to determine the effect of rice genotype and BPH treatment between susceptible and resistant rice varieties during the BPH infestation (Figure 3A-B). adaptations as illustrated in Figure 3. Day 1 block scores plot (ESI+ : PC1=14.8\% TEV and PC2 $=11.7 \%$ TEV; ESI- : PC1=12.6\% TEV and PC2=11.3\% TEV) showed slightly different profiles between susceptible KD and resistant IL308 varieties after the BPH infestation, whereas Day 4 block (ESI+ : PC1=61.0\% TEV and PC2=8.7\% TEV; ESI- : PC1=50.5\% TEV and $\mathrm{PC} 2=10.7 \%$ TEV) and Day 8 block (ESI+ : $\mathrm{PC} 1=27.0 \%$ TEV and $\mathrm{PC} 2=26.6 \%$ TEV, ESI- : PC1=50.5\% TEV and PC2=10.7\% TEV) scores plots showed more dramatic differences between the susceptible KD and the resistant IL308 varieties within the BPHinfested groups. The results clearly revealed different metabolic responses to the BPH infestation between the susceptible and the resistant varieties. By contrast, the profiles in the control groups were similar in all the time blocks, indicating similar metabolic states of the two groups.

To identify key metabolites with significant changes, the processed data were subsequently analyzed according to the data analysis workflow and summarized results shown in Figure 1. Three-way ANOVA indicated that a total of 801 features in ESI+ and 444 features in ESI- significantly contributing to these differences; i.e., metabolite features with 
adjusted $p$-value $<0.05$ after FDR $<0.001$ at least genotype factor, two factors by

genotype/treatment and genotype/time, and multiple factors by genotype/treatment/time were selected to be significantly different (Table S1). Of these, cluster analysis revealed that a total of 40 metabolite features in ESI+ and 56 metabolite features in ESI- could be grouped into five different alteration patterns, followed by Mann-Whitney U test for control versus BPH-infested groups per genotype per time. This allows elucidation of significant metabolites associated with the BPH infestation (Table S2). Subsequently, the significant metabolite features (a total of 9 features from ESI+ and 18 features from ESI-) were tentatively identified (Table S3).

\section{Alteration patterns and significant metabolites affected by BPH infestation}

Different patterns were observed in the clustergram in both profiles obtained from ESI+ (Figure 4A) and from ESI- (Figure 4B), providing diverse range of metabolite features that were changes in the plant host in response to the $\mathrm{BPH}$ infestation. While there was a common response found in both rice varieties, there were also different responses between the susceptible and the resistant rice, particularly with different response times after the infestation. The susceptible rice showed induced response at the latter time points (Days 4 and 8), whereas the resistant rice showed both constitutive and induced responses at the early timepoint (Day 1) and at the late timepoint (Day 8) (Figure 4A-B). Of these, three major responses were observed in Figures $4 \mathrm{~A}$ and $4 \mathrm{~B}$ and these alteration patterns are described below:

First, the common stress response (Cluster I of both ESI modes: ESI+ (9 features) 
357

in the treatment groups. Among these metabolite features, four $\left(3.44 \_477.1210 \mathrm{~m} / \mathrm{z}\right.$, 8.98_688.2016n, 8.98_657.1760m/z and 9.17_717.1974m/z) were found to be significantly changed. Given their common pattern in both rice varieties, they might be involved in a core metabolic activity essentially for growth and/or involved in a stress response which might not directly be associated with the resistance process.

Second, the unique response in the susceptible KD variety (Cluster IIa, IIb and IIc of ESI+: 7, 6 and 13 features, respectively and Cluster II of ESI-: 14 features) represented metabolites whose levels were increased only in the susceptible variety at late stage (Days 4 and 8) of the infestation. Nine of these metabolite features $\left(1.34 \_169.0361 \mathrm{~m} / \mathrm{z}\right.$, 2.47_137.0478m/z, 3.29_228.0502m/z, 4.31_277.1655m/z, 5.47_243.1346m/z, 5.95_272.1731n, 6.09_274.1205m/z, 9.24_571.1976m/z and 9.25_537.2135m/z) were found to be significantly changed. Of the nine features, three metabolites were identified as uric acid (Level 2 of MSI, 4 and 2.2 fold increase), hypoxanthine (Level 1 of MSI, 6 and 5.6 fold increase) and 4-(L-Alanin-3-yl)-2-hydroxy-cis,cis-muconate 6-semialdehyde (Level 2 of MSI, 1.5 and 2.7 fold increase) (Table S3), which belong to purine and amino acid metabolism. These late induction patterns after the infestation suggested that these metabolites might not be effective biomolecules for defense against the insect. However, the alteration patterns might be related to the hopper burn symptom in the susceptible variety, suggesting that these metabolite changes may be associated with cell death or the biotic stress response. These metabolites might be used as markers of the progression of hopper burn.

Third, the unique response in the resistant IL308 rice variety (Cluster III of ESI+ and ESI-) represented three different metabolite alteration patterns whose levels were significantly higher than those in the susceptible variety as in the following subgroups: 
The first subgroup, constitutive response (Cluster IIIa of ESI-: 9 features) represented metabolites whose levels were significantly higher than those in the susceptible variety in both before and after the infestation. Three significantly changed metabolite features $\left(9.73 \_515.1185 \mathrm{~m} / \mathrm{z}, 7.97 \_577.1516 \mathrm{~m} / \mathrm{z}\right.$ and $\left.11.38 \_583.3074 \mathrm{~m} / \mathrm{z}\right)$ were identified as dicaffeoylquinic acid (Level 1 of MSI, 4.7 fold increase), and 20-hydroxyecdysone acetate (Level 2 of MSI, 2.5 fold increase) which belong to phenolic acid, flavonoid, and terpenoid compound classes, respectively. Given their constitutive and unique patterns in resistant variety, these metabolites might be important as constitutive specialized (secondary) metabolites to protect against the insect attack.

The second subgroup, early induction at Day1 post-infestation (Cluster III of ESI+: 5 features and Cluster IIIb of ESI-: 12 features) represented metabolites whose levels were significantly increased only in the resistant variety at Day1 post-infestation. Five metabolites (7.25_564.1431n, 7.40_564.1431n, 7.50_757.1970m/z, 7.80_533.1254m/z, 7.97_577.1516m/z and 8.21_565.1520m/z) were found to be significant different, and identified (Level 1 or 2; see Table S3) as iso-schaftoside (1.7 fold increase), schaftoside (1.8 fold increase), rhoifolin (Level 2 of MSI, 3.4 fold increase), apigenin-6-C-glucoside-8-Carabinoside, cyanidin 3-O-(6-O-p-coumaroyl) glucoside-5-O-glucoside, apigenin 6-C- $\alpha-\mathrm{L}-$ arabinoside-8-C- $\beta$-L-arabinoside and naringenin 8 -C- $\alpha$-L-arabinopyranosyl-7-O- $\beta$-Dglucopyranoside belonging to flavonoid glycosides. These metabolites in this alteration pattern might be involved in inducible defense against the insect attack.

The third subgroup, late induction at Day8 post-infestation (Cluster IIIc of ESI-: 7 features) represented metabolites whose levels increased only in the resistant variety at Day8 postinfestation. Three significant metabolite features (8.36_245.1383m/z, 12.36_241.1430m/z and $12.52 \_227.1638 \mathrm{~m} / \mathrm{z}$ ) were tentatively identified as 3-hydroxydodecanedioic acid, 2,4- 
dioxotridecanoic acid and 12-oxotridecanoic acid (all Level 2 of MSI) belonging to fatty acid class. However, there were no scientific evidences about these fatty acids in plants and other organisms. Based on the increasing levels of these metabolites at late stage of the infestation, they might not directly be associated with the resistance processes.

To evaluate the identified metabolites further, metabolite levels for each of the nine significant metabolites were considered (Figure 5). First, three metabolites with significant difference $(p<0.05)$ in the susceptible rice variety (uric acid, hypoxanthine and 4-(alanine-3yl)-2-hydroxy-muconate semialdehyde) were found to be similar in the control groups in both rice varieties, whereas those in the BPH-treated groups were different between KD and IL308 (Figure 5A). Specifically, uric acid levels in the KD were significantly increased by Day1 to Day 8 after the BPH treatment whereas those in the IL308 were increased by Day 4 to Day 8 which was slower response than in the KD. Hypoxanthine levels in the KD and the IL308 were significantly increased by Day 1 to Day 4 after the treatment; however, by Day 8, the hypoxanthine level was still significantly increased in the KD whereas those in the IL308 was significantly decreased $(p<0.05)$. The levels of 4-(alanine-3-yl)-2-hydroxy-muconate semialdehyde were significantly increased in the KD from Day 4 to Day 8 after the infestation whereas those in the IL308 were not changed. Hypoxanthine and uric acid are involved in purine metabolism, and 4-(alanine-3-yl)-2-hydroxy-muconate semialdehyde is involved in tyrosine metabolism, suggesting that the KD variety employed primary metabolism important for its growth in response to the biotic stress.

By contrast, schaftoside, isoschaftoside, rhoifolin and apigenin- $6-\alpha-$ arabinoside- 8 $\beta$-arabinoside metabolites whose levels were significantly increased $(p<0.05)$ by $1.8,1.7,3.4$ and 1.6 fold increase, respectively, only in the resistant IL308 variety by Day 1 postinfestation, decreased in susceptible variety (Figure 5B). Flavonoid glycosides are part of the 
flavonoid family which has previously been reported to play role on insect resistance in several crops such as sorghum, sunflower, eucalyptus (Tenango et al., 2017), wheat (Dreyer and Jones, 1981) and maize (Mierziak et al., 2014). Therefore, these metabolites may also be involved in $\mathrm{BPH}$ resistance in rice.

432 metabolites levels were constitutively higher (4.7 and 2.5 fold increase, respectively) than 433 those in the susceptible variety throughout the experiment (Figure 5C). After the BPH 434 infestation, the three metabolites had different patterns. Particularly, dicaffeoylquinic acid 435 was significantly increased $(p<0.05)$ by BPH at Day 4 and decreased at Day 8 as compared to 436 control treatment, whereas 20-hydroxyecdysone acetate was significantly increased $(p<0.05)$ 437 at Day 1 and decreased at Day 8. These specialized metabolites are involved in 438 phenylpropanoid, flavonoid or terpenoid metabolism that may play important roles on BPH 439 resistance.

\section{Discussion}

441 Different phenotypic responses between susceptible and resistance rice varieties upon $442 \quad$ BPH treatment 
undertaken. After chemometric analyses (viz. MBPCA and univariate statistical testing) significant metabolites and metabolic pathways were revealed that were different between susceptible and resistance varieties during BPH infestation, which has provided key information for metabolic adaptation of plant as defense against BPH attack (Figure 6).

\section{Metabolic response of the susceptible rice during the BPH infestation}

Hypoxanthine and uric acid levels were found in the infested susceptible rice variety in higher levels than in the control and the resistant variety. These results agree with our previous study using ${ }^{1} \mathrm{H}-\mathrm{NMR}$-based metabolomics comparing metabolomics responses in susceptible rice variety (KD) between $\mathrm{BPH}$ treatment and control (no BPH treatment) at Day 1, 4 and 8 and revealing that hypoxanthine level was affected in the BPH-treated group at the late time points (Day 4 and 8) (Uawisetwathana et al., 2015). These metabolites are found to be involved in purine metabolism via a salvage pathway (Ashihara et al., 2018). In the rice plant, hypoxanthine is reversibly synthesized by inosine to produce xanthine and consequently convert to uric acid. The purine salvage pathway in plants is generally employed for remobilization of nitrogen and nutrient recycling which saves energy usage (Brychkova et al., 2008). Uric acid is then finally catabolized to ammonia. The accumulation of ammonia causes cytotoxicity in plants (Hauck et al., 2014). Hypoxanthine is a prime metabolite contributing to responses to environmental and biotic stresses (Pastor et al., 2014).

From this study and literature, it could be hypothesized that BPH-susceptible rice adapts itself by utilizing hypoxanthine and uric acid through salvage pathway of purine biosynthesis for its survival.

In summary, it is proposed that the susceptible rice responded to the insect attack by increased turnover of nucleotide synthesis via a salvage pathway to acclimate and maintain cellular function close to normal conditions. However, in this study, there were no secondary 
metabolites that were significantly correlating to BPH-defense processes in this variety. It could be suggested that, apart from adaptation process, there is lack of systemic defense processes in the susceptible rice.

\section{Metabolic response of the resistant rice during the BPH infestation}

Interestingly, this study also found flavonoid glycoside (MSI level 1), chlorogenic acid (MSI level 1) and hydroxytriterpenoid (MSI level 2) in the resistant rice variety. With hydrophilic as their functional groups, these compounds are water soluble as reported in these studies (Matsui et al., 2004, Slámová et al., 2018) which could be extracted by

methanol/water used in this study. Flavonoid glycosides are major plant secondary metabolites composed of glycosylated flavones, flavonols, anthocyanins, flavanones, isoflavones and etcetera which play essential roles in plants under stress conditions such as UVB screening, pollination, seed development, allellopathy and plant-pathogen interaction (Harborne and Williams, 2000, Falcone Ferreyra et al., 2012). Flavonoid glycosides are diverse across plant species. Thus, it is necessary to know flavonoid glycosides specific to rice, particularly in response to the $\mathrm{BPH}$ attack. In this study, several flavonoid glycosides were found to be significantly elevated in the post-infested resistant rice and these included schaftoside (apigenin 6-C- $\beta$-D-glucopyranosyl-8-C- $\alpha$-L-arabinopyranoside), iso-schaftoside (apigenin 6-C- $\alpha$-L-arabinopyranoside-8-C- $\beta$-D-glucopyranoside), rhoifolin (apigenin 7-Oneohesperidoside) and apigenin 6-C- $\alpha-\mathrm{L}$-arabinopyranosyl-8-C- $\beta$-L-arabinopyranoside. Previous reports showed that schaftoside has been found in rice phloem sap as a BPH sucking deterrent in the Rathu Heenati cultivar (Stevenson et al., 1996) and IR 62 variety (Grayer et al., 1994). The mechanism of action of schaftoside was reported to inhibit $C D K 1$ of the BPH, causing the insect cell death (Hao et al., 2018), and has been shown to have nematocidal activity against a plant parasite Meloidogyne incogtina in the tuber species Arisaema erubescens (Du et al., 2011). Iso-schaftoside has been reported to have an inhibitory effect in 
a tick clover (Desmodium spp.) to a witchweed (Striga spp.) interaction (Hamilton et al.,

2012). There is currently no disease resistance evidence for apigenin $6-\mathrm{C}-\alpha-\mathrm{L}-$

arabinopyranosyl-8-C- $\beta$-L-arabinopyranoside. Rhoifolin, apigenin 7-O-neohesperidoside, is

a member of apigenin glycosides found in various plants such as bitter orange, bergamot, lemon, tomatoes, artichoke, banana, grape and grapefruit (Refaat et al., 2015, Gattuso et al., 2006, Gattuso et al., 2007). It has been reported to provide antioxidant, anti-inflammatory, antimicrobial and anticancer benefits to human's health (Refaat et al., 2015). In citrus plants, rhoifolin accumulation has been found to be associated with drought-tolerant condition (Santos et al., 2017). Thus, the present study revealed that the flavonoid aglycones, apigenin, was found to be major in rice leave during $\mathrm{BPH}$ infestation and the early induction of apigenin glycosides after the insect attack compared to the susceptible KD variety suggests that these metabolites may play an important role in inducible defense mechanism.

Interestingly, another response found in the resistant IL308 variety was constitutive elevation of several metabolites compared to the susceptible KD variety. The metabolites in this response contained dicaffeoylquinic acid and 20-hydroxyecdysone acetate. With regards to the plant defense against herbivores, constitutive defenses such as thorn, trichrome and chemical toxins indicate a promising plant defense mechanism as these chemicals are produced at all developmental stages and have durable broad spectrum of resistance (Kessler and Baldwin, 2002, Wittstock and Gershenzon, 2002, War et al., 2012). This will be expanded as follows:

Firstly, 3,4-di- $O$-caffeoylquinic acid is one of secondary metabolites from phenylpropanoid pathway derived by $p$-coumaric acid in combination with quinic acid. Its product is a chlorogenic acid, called caffeoylquinic acid which is a precursor to produce dicaffeoylquinic acid (Moglia et al., 2014, Clifford et al., 2017). It has been reported to have allelopathic effect to inhibit fungal (Fusarium. solani) growth in sweet potato (Harrison et al., 
2008), to have a protective effect against oxidative damage from salinity in tomatoes

(Martinez et al., 2016), as well as ultraviolet radiation protection in artichoke leaf (Moglia et al., 2008), has particularly protective effect against predators in tomatoes such as fruitworm Heliothis zea and beet armyworm Spodoptera exigua (Clifford et al., 2017, Felton et al., 1989, Kundu and Vadassery, 2019), and synthetic dicinnamoyl 4-deoxy quinic acid and its methyl ester derivatives were shown to have toxicity effect against pea aphid Acrythosiphon pisum (Li et al., 2016). The mode of action has been proposed that quinones derived from dicaffeoylquinic acid can react with nucleophiles and link covalently to insect protein; subsequently limit the growth of herbivores (Kuijpers et al., 2012, Pierpoint, 1969, Felton and Duffey, 1990). Caffeoylquinic acid is a common polyphenolic compound found in rice; however, this is the first report where a 3,4-di-O-caffeoylquinic acid has been detected in rice with potential insect resistance. Hence, in this study, a higher amount of 3,4-di- $O$ caffeoylquinic acid in the resistant IL308 rice variety, than in the susceptible KD, might suggest its protective function against the $\mathrm{BPH}$ attack.

Secondly, 20-hydroxyecdysone acetate is a phytoecdysteroid synthesized by plants for defense against phytophagous insects (Adler and Grebenok, 1999, Dinan, 2001). The chemical structures are an analogue to the insect molting hormone, ecdysteroids, which is essential during the molting process of the insect, thus these compounds have potential role on inhibiting insect growth (Abubakirov, 1980). 20-hydroxyecdysone is synthesized from mevalonic acid in the mevalonate pathway of the plants cell using acetyl-CoA as a precursor. Under insect herbivores damage and wound induction, 20-hydroxyecdysone was found to be accumulated (Schmelz et al., 2002). 20-hydroxyecdysone showed antitumor, antibacterial and antioxidant from Silene plants (Baltayev, 1998, Csábi et al., 2016) as well as insect growth regulator in fern, purposing to be replacing synthetic insecticides for insect pest management programs (Chaubey, 2018). From its linkage to inhibit insect growth, we hypothesize that 
20-hydroxyecdysone acetate might involve as protective metabolite in rice after the BPH infestation. Not only was 20-hydroxyecdysone acetate constitutively increased in the resistant IL308 compared to the susceptible KD variety, but it was also induced by the BPH at the early stage of infestation (Day1).

In combination these results may suggest that the resistant rice variety has orchestrated several defense mechanisms using a series of metabolites related in phenylpropanoid, flavonoid or terpenoid pathways for both constitutive and inducible defenses. Furthermore, the adaptation process found in the resistant variety in the early stage of infestation resulted in the induction of increased hypoxanthine level on Day 1.

\section{Concluding Remarks}

This study demonstrated the applicability of UPLC-QToF-MS combined with MBPCA to identify metabolites that were differential between BPH-susceptible KD and its BPH-resistant IL308 isogenic line, upon normal (pest free) and BPH infestation conditions.

Different metabolic responses were discovered with significant metabolites contributing to the responses between the susceptible and the resistant rice varieties being highlighted and these differential metabolites were mapped onto plant metabolite pathways. Focusing on the resistant variety, the significantly altered metabolites post-infestation were involved in phenylpropanoids, flavonoids and terpenoids metabolism. These secondary metabolites may potentially act as constitutive and inducible defenses against the $\mathrm{BPH}$ attack. The constitutive metabolites could be used as metabolite markers for the isogenic line selection without brown planthopper induction to accelerate precision breeding program. Furthermore, these bioactive metabolites could be applied by spraying onto susceptible rice plants to protect against BPH attack. In conclusion, this study explored potential specialized metabolites to defend against 
574 the insect herbivore, brown planthopper, and can be useful for plant breeding program to

575 select insect resistant plants without induction of elicitor.

\section{Acknowledgments}

578 This work was financially supported by Platform Technology Program (P-12-01893, 579 P-16-50339 and P-18-50973), National Center for Genetic Engineering and Biotechnology 580 (BIOTEC, Thailand). The PhD scholarship to Umaporn Uawisetwathana was awarded from 581 Graduate and Professional Development Division, National Science and Technology 582 Development Agency (NSTDA, Thailand).

\section{Author Contribution Statement}

584 NK, TT, AV, CE and RG conceived and designed research. WK and UU conducted 585 experiments. UU, OPC contributed analytical tools. UU and YX analyzed data. UU, NK, IN, 586 RG, YX, OPC, CE and TP wrote the manuscript. All authors read and approved the 587 manuscript.

\section{Competing interests}

589 The authors declare that they have no competing interests.

\section{Compliance with ethical requirement}

591 This article does not contain any studies with human subjects.

\section{Figure Legends}

593 Figure 1. MS data processing and analysis workflow in both ESI+ and ESI- modes. 
Figure 2. Physiological response between control (-BPH) and treatment (+BPH) by Days 1, 4 and 8 for susceptible KD and resistant IL308 rice varieties ( $\mathrm{n}=3$ /group). Red arrow indicates hopper burn symptom in the BPH-susceptible $\mathrm{KD}$ variety.

Figure 3. MBPCA time block scores plots between control $(-\mathrm{BPH})$ and treatment $(+\mathrm{BPH})$ of susceptible KD and resistant IL308 rice varieties. A) Day 1, 4 and 8 scores plots obtained from ESI+ (4,688 variables) and B) Day 1, 4 and 8 scores plots obtained from ESI- $(2,151$ variables).

Figure 4. Clustergram analysis of susceptible KD and resistant IL308 rice varieties between control $(-\mathrm{BPH})$ and treatment $(+\mathrm{BPH})$ by Days 1,4 and 8 . A) Clustergrams obtained from 801 metabolite features of ESI+ showing five different alteration patterns (40 metabolite features) with their level changes greater than 1.5 folds and B) Clustergrams obtained from 444 metabolite features of ESI- showing five different alteration patterns (56 metabolite features) with their level changes greater than 1.5 folds.

Figure 5. Significant metabolites affected by $\mathrm{BPH}$ infestation ( $\mathrm{n}=3$ /group). A) three metabolites showing late induction in the susceptible KD variety, B) four metabolites showing early induction in the resistant IL308 variety and C) two metabolites showing constitutive in the resistant IL308 variety. Mann-Whitney U test was used to compare the control (solid line) and the treatment (dashed line) groups $\left({ }^{*} p\right.$-value $<0.05,{ }^{*} p$-value $<0.01$ and $* * * p$-value $<0.001)$ per time per genotype.

Figure 6. Proposed metabolic pathways affected in BPH-susceptible and BPH-resistant rice varieties in response to $\mathrm{BPH}$ infestation

\section{Supplementary data}

Supplementary Figure 1. Experimental design in this study. Paddy soil was dug from the field and put in the pots. Rice seeds were germinated in the pots for one week before water was added into the pot to cover the soil. Ten gram of nitrogen fertilizer (46-0-0) was added to 
619 each pot at the 3-week-old seedling stage. The water was always maintained to cover the soil 620 during the experiment.

621 Supplementary Figure 2. Base peak intensity (BPI) chromatogram of leaf extracts from 622 BPH-susceptible KD (A) and BPH-resistant IL308 (B) at Day 1 by UPLC-QToF-MS analysis 623 in ESI+ and ESI-. $-\mathrm{BPH}=$ control group and $+\mathrm{BPH}=$ treatment group.

624 Supplementary Figure 3. Principal component analysis (PCA) scores plots of rice BPH625 resistant traits including with quality control samples to show reproducibility. A) PCA scores 626 plot obtained from ESI+ and B) PCA scores plot obtained from ESI-.

627 Supplementary Figure 4. MBPCA Super scores of rice BPH-resistant traits. Diamond 628 symbol represents BPH-susceptible KD variety and circle symbol represents BPH-resistant 629 IL308 variety. Clear symbol represents the samples in control group and solid symbol 630 represents the samples in treatment group. Blue, green and red colors represent days 1, 4 and 6318 , respectively.

632 Supplementary Table 1. Significant metabolite features selected by three-way ANOVA ( $p$ 633 value $<0.05)$ based on genotype factor and its interactions (time and treatment). A sheet 634 "Supplementary Table 1_Pos" represents the significant metabolite features obtained from 635 ESI+ (801 features) and a sheet "Supplementary Table 1 Neg" represents the significant 636 metabolite features obtained from ESI- (444 features) modes. Significant metabolite features 637 labelled in red color with bold letter showed obvious alteration patterns in cluster analysis.

638 Supplementary Table 2. Mean \pm SD ( $n=3$ X3/group) of metabolite features based on their 639 alteration pattern with level changes $>1.5$ folds were analyzed by Mann-Whitney $U$ test to 640 select significant differences between control and treatment. A sheet "Supplementary Table 641 2_Pos" represents 40 metabolite features and a sheet "Supplementary Table 2_Neg" 642 represents 56 metabolite features. 
Supplementary Table 3. Summary of the metabolite annotation and identification of the

significant metabolite features

\section{References}

Abubakirov, N.K. (1980). New Phytoecdysones. IN Ananchenko, S.N. (Ed.) Frontiers of Bioorganic Chemistry and Molecular Biology. Pergamon.

Adler, J.H. \& Grebenok, R.J. (1999). Occurrence, Biosynthesis, and Putative Role of Ecdysteroids in Plants. Critical Reviews in Biochemistry and Molecular Biology, 34: 253-264. DOI 10.1080/10409239991209282

Allwood, J.W. \& Goodacre, R. (2010). An introduction to liquid chromatography-mass spectrometry instrumentation applied in plant metabolomic analyses. Phytochemical Analysis, 21: 33-47. DOI 10.1002/pca.1187

Allwood, J.W., Woznicki, T.L., Xu, Y., Foito, A., Aaby, K., Sungurtas, J., Freitag, S., Goodacre, R., Stewart, D., Remberg, S.F., Heide, O.M. \& Sønsteby, A. (2019). Application of HPLC-PDA-MS metabolite profiling to investigate the effect of growth temperature and day length on blackcurrant fruit. Metabolomics, 15: 12. DOI 10.1007/s11306-018-1462-5

Ashihara, H., Stasolla, C., Fujimura, T. \& Crozier, A. (2018). Purine salvage in plants. Phytochemistry, 147: 89-124. DOI https://doi.org/10.1016/j.phytochem.2017.12.008

Balmer, D., Flors, V., Glauser, G. \& Mauch-Mani, B. (2013). Metabolomics of cereals under biotic stress: current knowledge and techniques. Frontiers in Plant Science, 4. DOI 10.3389/fpls.2013.00082

Baltayev, U.A. (1998). Ecdysteroside, a phytoecdysteroid from Silene tatarica. Phytochemistry, 47: 1233-1235. DOI https://doi.org/10.1016/S0031-9422(97)00715-2

Biais, B., Allwood, J.W., Deborde, C., Xu, Y., Maucourt, M., Beauvoit, B., Dunn, W.B., Jacob, D., Goodacre, R., Rolin, D. \& Moing, A. (2009). 1H NMR, GC-EI-TOFMS, and Data Set Correlation for Fruit Metabolomics: Application to Spatial Metabolite Analysis in Melon. Analytical Chemistry, 81: 2884-2894. DOI 10.1021/ac9001996

Broadhurst, D., Goodacre, R., Reinke, S.N., Kuligowski, J., Wilson, I.D., Lewis, M.R. \& Dunn, W.B. (2018). Guidelines and considerations for the use of system suitability and quality control samples in mass spectrometry assays applied in untargeted clinical metabolomic studies. Metabolomics : Official journal of the Metabolomic Society, 14: 72-72. DOI 10.1007/s11306-018-1367-3

Brychkova, G., Fluhr, R. \& Sagi, M. (2008). Formation of xanthine and the use of purine metabolites as a nitrogen source in Arabidopsis plants. Plant Signaling \& Behavior, 3: 999-1001. DOI 10.4161/psb.6304

Carrizo, D., Chevallier, O.P., Woodside, J.V., Brennan, S.F., Cantwell, M.M., Cuskelly, G. \& Elliott, C.T. (2017). Untargeted metabolomic analysis of human serum samples associated with exposure levels of Persistent organic pollutants indicate important perturbations in Sphingolipids and Glycerophospholipids levels. Chemosphere, 168: 731-738. DOI https://doi.org/10.1016/j.chemosphere.2016.11.001

Chaubey, M.K. (2018). Role of Phytoecdysteroids in Insect Pest Management: A Review. Journal of Agronomy, 17: 1-10.

Clifford, Michael n., Jaganath, I.B., Ludwig, I.A. \& Crozier, A. (2017). Chlorogenic acids and the acyl-quinic acids: discovery, biosynthesis, bioavailability and bioactivity. Natural Product Reports, 34: 1391-1421. DOI 10.1039/C7NP00030H 
Csábi, J., Martins, A., Sinka, I., Csorba, A., Molnár, J., Zupkó, I., Tóth, G., Tillekeratne, L.M.V. \& Hunyadi, A. (2016). Synthesis and in vitro evaluation of the antitumor potential and chemo-sensitizing activity of fluorinated ecdysteroid derivatives. MedChemComm, 7: 2282-2289. DOI 10.1039/C6MD00431H

Dinan, L. (2001). Phytoecdysteroids: biological aspects. Phytochemistry, 57: 325-339. DOI https://doi.org/10.1016/S0031-9422(01)00078-4

Dreyer, D.L. \& Jones, K.C. (1981). Feeding deterrency of flavonoids and related phenolics towards Schizaphis graminum and Myzus persicae: Aphid feeding deterrents in wheat. Phytochemistry, 20: 2489-2493. DOI https://doi.org/10.1016/00319422(81)83078-6

Du, S.S., Zhang, H.M., Bai, C.Q., Wang, C.F., Liu, Q.Z., Liu, Z.L., Wang, Y.Y. \& Deng, Z.W. (2011). Nematocidal Flavone-C-Glycosides against the Root-Knot Nematode (Meloidogyne incognita) from Arisaema erubescens Tubers. Molecules, 16: 5079.

Dunn, W.B., Broadhurst, D., Begley, P., Zelena, E., Francis-Mcintyre, S., Anderson, N., Brown, M., Knowles, J.D., Halsall, A., Haselden, J.N., Nicholls, A.W., Wilson, I.D., Kell, D.B. \& Goodacre, R. (2011). Procedures for large-scale metabolic profiling of serum and plasma using gas chromatography and liquid chromatography coupled to mass spectrometry. Nat Protocols, 6: 1060-1083. DOI http://www.nature.com/nprot/journal/v6/n7/abs/nprot.2011.335.html\#supplementaryinformation

Eisen, M.B., Spellman, P.T., Brown, P.O. \& Botstein, D. (1998). Cluster analysis and display of genome-wide expression patterns. Proceedings of the National Academy of Sciences, 95: 14863-14868.

Falcone Ferreyra, M.L., Rius, S. \& Casati, P. (2012). Flavonoids: Biosynthesis, Biological functions and Biotechnological applications. Frontiers in Plant Science, 3. DOI 10.3389/fpls.2012.00222

Felton, G.W., Donato, K., Del Vecchio, R.J. \& Duffey, S.S. (1989). Activation of plant foliar oxidases by insect feeding reduces nutritive quality of foliage for noctuid herbivores. Journal of Chemical Ecology, 15: 2667-2694. DOI 10.1007/BF01014725

Felton, G.W. \& Duffey, S.S. (1990). Inactivation of baculovirus by quinones formed in insect-damaged plant tissues. Journal of Chemical Ecology, 16: 1221-1236. DOI 10.1007/bf01021021

Fujita, D., Kohli, A. \& Horgan, F.G. (2013). Rice Resistance to Planthoppers and Leafhoppers. Critical Reviews in Plant Sciences, 32: 162-191. DOI 10.1080/07352689.2012.735986

Gattuso, G., Barreca, D., Gargiulli, C., Leuzzi, U. \& Caristi, C. (2007). Flavonoid composition of Citrus juices. Molecules (Basel, Switzerland), 12: 1641-1673. DOI 10.3390/12081641

Gattuso, G., Caristi, C., Gargiulli, C., Bellocco, E., Toscano, G. \& Leuzzi, U. (2006). Flavonoid Glycosides in Bergamot Juice (Citrus bergamia Risso). Journal of Agricultural and Food Chemistry, 54: 3929-3935. DOI 10.1021/jf060348z

Grayer, R.J., Harborne, J.B., Kimmins, F.M., Stevenson, P.C. \& Wijayagunasekera, H.N.P. (1994). Phenolics in rice phloem sap as sucking deterrents to the brown planhopper, Nilapavata lugens. 381 ed.: International Society for Horticultural Science (ISHS), Leuven, Belgium.

Hamilton, M.L., Kuate, S.P., Brazier-Hicks, M., Caulfield, J.C., Rose, R., Edwards, R., Torto, B., Pickett, J.A. \& Hooper, A.M. (2012). Elucidation of the biosynthesis of the di-Cglycosylflavone isoschaftoside, an allelopathic component from Desmodium spp. that inhibits Striga spp. development. Phytochemistry, 84: 169-176. DOI http://dx.doi.org/10.1016/j.phytochem.2012.08.005 
Hao, P.-Y., Feng, Y.-L., Zhou, Y.-S., Song, X.-M., Li, H.-L., Ma, Y., Ye, C.-L. \& Yu, X.-P. (2018). Schaftoside Interacts With NICDK1 Protein: A Mechanism of Rice Resistance to Brown Planthopper, Nilaparvata lugens. Frontiers in Plant Science, 9. DOI 10.3389/fpls.2018.00710

Harborne, J.B. \& Williams, C.A. (2000). Advances in flavonoid research since 1992. Phytochemistry, 55: 481-504. DOI http://dx.doi.org/10.1016/S0031-9422(00)00235-1

Harrison, H., Mitchell, T.R., Peterson, J.K., Wechter, W.P., Majetich, G.F. \& Snook, M.E. (2008). Contents of Caffeoylquinic Acid Compounds in the Storage Roots of Sixteen Sweetpotato Genotypes and Their Potential Biological Activity. Journal of the American Society for Horticultural Science, 133.

Hauck, O.K., Scharnberg, J., Escobar, N.M., Wanner, G., Giavalisco, P. \& Witte, C.-P. (2014). Uric Acid Accumulation in an Arabidopsis Urate Oxidase Mutant Impairs Seedling Establishment by Blocking Peroxisome Maintenance. The Plant Cell, 26: 3090. DOI 10.1105/tpc.114.124008

Heinrichs, E.A. (1979). Brown Planthopper: Threat to Rice Production in Asia. IN A., H.E. (Ed.) Chemical control of the brown planthopper. Philippines: International Rice Research Institute.

Heong, K.L.a.H., B. (2009). Planthopper: New treats to the sustainability of intensive rice production systems in Asia. Los Banos (Philippines): International Rice Research Institute.

Hu, J., Xiao, C. \& He, Y. (2016). Recent progress on the genetics and molecular breeding of brown planthopper resistance in rice. Rice (New York, NY), 9: 30-30. DOI 10.1186/s12284-016-0099-0

Jairin, J., Teangdeerith, S., Leelagud, P., Kothcharerk, J., Sansen, K., Yi, M., Vanavichit, A. \& Toojinda, T. (2009). Development of rice introgression lines with brown planthopper resistance and KDML105 grain quality characteristics through markerassisted selection. Field Crops Research, 110: 263-271. DOI http://dx.doi.org/10.1016/j.fcr.2008.09.009

Kamolsukyeunyong, W., Ruengphayak, S., Chumwong, P., Kusumawati, L., Chaichoompu, E., Jamboonsri, W., Saensuk, C., Phoonsiri, K., Toojinda, T. \& Vanavichit, A. (2019). Identification of spontaneous mutation for broad-spectrum brown planthopper resistance in a large, long-term fast neutron mutagenized rice population. Rice, 12: 126. DOI 10.1186/s12284-019-0274-1

Kamolsukyunyong, W., Sukhaket, W., Ruanjaichon, V., Toojinda, T. \& Vanavichit, A. (2013). Single-feature polymorphism mapping of isogenic rice lines identifies the influence of terpene synthase on brown planthopper feeding preferences. Rice, 6: 18 .

Kang, K., Yue, L., Xia, X., Liu, K. \& Zhang, W. (2019). Comparative metabolomics analysis of different resistant rice varieties in response to the brown planthopper Nilaparvata lugens Hemiptera: Delphacidae. Metabolomics, 15: 62. DOI 10.1007/s11306-0191523-4

Kessler, A. \& Baldwin, I.T. (2002). Plant responses to insect herbivory: The emerging molecular analysis. Annual Review of Plant Biology, 53. DOI 10.1146/annurev.arplant.53.100301.135207

Kuijpers, T.F.M., Narváez-Cuenca, C.-E., Vincken, J.-P., Verloop, A.J.W., Van Berkel, W.J.H. \& Gruppen, H. (2012). Inhibition of Enzymatic Browning of Chlorogenic Acid by Sulfur-Containing Compounds. Journal of Agricultural and Food Chemistry, 60: 3507-3514. DOI 10.1021/jf205290w

Kundu, A. \& Vadassery, J. (2019). Chlorogenic acid-mediated chemical defence of plants against insect herbivores. Plant Biology, 21: 185-189. DOI 10.1111/plb.12947 
Kusumawati, L., Chumwong, P., Jamboonsri, W., Wanchana, S., Siangliw, J.L., Siangliw, M., Khanthong, S., Vanavichit, A., Kamolsukyeunyong, W. \& Toojinda, T. (2018). Candidate genes and molecular markers associated with brown planthopper (Nilaparvata lugens Stål) resistance in rice cultivar Rathu Heenati. Mol Breeding, 38: 88. DOI 10.1007/s11032-018-0847-5

Li, X., Grand, L., Pouleriguen, T., Queneau, Y., Da Silva, P., Rahbé, Y., Poëssel, J.-L. \& Moebs-Sanchez, S. (2016). Synthesis of new dicinnamoyl 4-deoxy quinic acid and methyl ester derivatives and evaluation of the toxicity against the pea aphid Acyrthosiphon pisum. Organic \& Biomolecular Chemistry, 14: 2487-2497. DOI 10.1039/C5OB02483H

Li, Z., Xue, Y., Zhou, H., Li, Y., Usman, B., Jiao, X., Wang, X., Liu, F., Qin, B., Li, R. \& Qiu, Y. (2019). High-resolution mapping and breeding application of a novel brown planthopper resistance gene derived from wild rice (Oryza. rufipogon Griff). Rice, 12: 41. DOI 10.1186/s12284-019-0289-7

Liu, C., Du, B., Hao, F., Lei, H., Wan, Q., He, G., Wang, Y. \& Tang, H. (2017). Dynamic metabolic responses of brown planthoppers towards susceptible and resistant rice plants. Plant biotechnology journal, 15: 1346-1357. DOI 10.1111/pbi.12721

Liu, C., Hao, F., Hu, J., Zhang, W., Wan, L., Zhu, L., Tang, H. \& He, G. (2010). Revealing Different Systems Responses to Brown Planthopper Infestation for Pest Susceptible and Resistant Rice Plants with the Combined Metabonomic and Gene-Expression Analysis. Journal of Proteome Research, 9: 6774-6785. DOI 10.1021/pr100970q

Liu, Y., Wu, H., Chen, H., Liu, Y., He, J., Kang, H., Sun, Z., Pan, G., Wang, Q., Hu, J., Zhou, F., Zhou, K., Zheng, X., Ren, Y., Chen, L., Wang, Y., Zhao, Z., Lin, Q., Wu, F., Zhang, X., Guo, X., Cheng, X., Jiang, L., Wu, C., Wang, H. \& Wan, J. (2015). A gene cluster encoding lectin receptor kinases confers broad-spectrum and durable insect resistance in rice. Nat Biotech, 33: 301-305. DOI 10.1038/nbt.3069

http://www.nature.com/nbt/journal/v33/n3/abs/nbt.3069.html\#supplementary-information Martinez, V., Mestre, T.C., Rubio, F., Girones-Vilaplana, A., Moreno, D.A., Mittler, R. \& Rivero, R.M. (2016). Accumulation of Flavonols over Hydroxycinnamic Acids Favors Oxidative Damage Protection under Abiotic Stress. Frontiers in plant science, 7: 838-838. DOI 10.3389/fpls.2016.00838

Matsui, T., Ebuchi, S., Fujise, T., Abesundara, K.J.M., Doi, S., Yamada, H. \& Matsumoto, K. (2004). Strong Antihyperglycemic Effects of Water-Soluble Fraction of Brazilian Propolis and Its Bioactive Constituent, 3,4,5-Tri-<i $>\mathrm{O}</ \mathrm{i}>$-caffeoylquinic Acid. Biological and Pharmaceutical Bulletin, 27: 1797-1803. DOI 10.1248/bpb.27.1797

Mierziak, J., Kostyn, K. \& Kulma, A. (2014). Flavonoids as Important Molecules of Plant Interactions with the Environment. Molecules, 19. DOI 10.3390/molecules191016240

Moglia, A., Lanteri, S., Comino, C., Acquadro, A., De Vos, R. \& Beekwilder, J. (2008). Stress-Induced Biosynthesis of Dicaffeoylquinic Acids in Globe Artichoke. Journal of Agricultural and Food Chemistry, 56: 8641-8649. DOI 10.1021/jf801653w Moglia, A., Lanteri, S., Comino, C., Hill, L., Knevitt, D., Cagliero, C., Rubiolo, P., Bornemann, S. \& Martin, C. (2014). Dual catalytic activity of hydroxycinnamoylcoenzyme A quinate transferase from tomato allows it to moonlight in the synthesis of both mono- and dicaffeoylquinic acids. Plant physiology, 166: 1777-1787. DOI 10.1104/pp.114.251371

Nakabayashi, R. \& Saito, K. (2015). Integrated metabolomics for abiotic stress responses in plants. Current Opinion in Plant Biology, 24: 10-16. DOI https://doi.org/10.1016/j.pbi.2015.01.003 
O'kane, A.A., Chevallier, O.P., Graham, S.F., Elliott, C.T. \& Mooney, M.H. (2013). Metabolomic Profiling of In Vivo Plasma Responses to Dioxin-Associated Dietary Contaminant Exposure in Rats: Implications for Identification of Sources of Animal and Human Exposure. Environmental Science \& Technology, 47: 5409-5418. DOI $10.1021 / \mathrm{es} 305345 \mathrm{u}$

Pastor, V., Balmer, A., Gamir, J., Flors, V. \& Mauch-Mani, B. (2014). Preparing to fight back: generation and storage of priming compounds. Frontiers in Plant Science, 5. DOI 10.3389/fpls.2014.00295

Pierpoint, W.S. (1969). O-Quinones formed in plant extracts. Their reaction with bovine serum albumin. Biochemical Journal, 112: 619. DOI 10.1042/bj1120619

Pitija, K., Kamolsukyumyong, W., Vanavichit, A., Sookwong, P. \& Mahatheeranont, S. (2014). Monoterpenoid Allelochemicals in Resistance Rice Varieties against Brown Planthoppers, Nilaparvata Lugens (Stål). Journal of Advanced Agricultural Technologies, 1: 7. DOI 10.12720/joaat.1.2.82-88

Refaat, J., Desoukey, S.Y., Ramadan, M.A. \& Kamel, M.S. (2015). RHOIFOLIN: A REVIEW OF SOURCES AND BIOLOGICAL ACTIVITIES. International Journal of Pharmacognosy, 2.

Ren, J., Gao, F., Wu, X., Lu, X., Zeng, L., Lv, J., Su, X., Luo, H. \& Ren, G. (2016). Bph32, a novel gene encoding an unknown SCR domain-containing protein, confers resistance against the brown planthopper in rice. Scientific reports, 6: 37645-37645. DOI 10.1038/srep37645

Santos, I.C.D., Almeida, A.-a.F.D., Pirovani, C.P., Costa, M.G.C., Silva, M.F.D.G.F.D., Bellete, B.S., Freschi, L., Soares Filho, W., Coelho Filho, M.A. \& Gesteira, A.D.S. (2017). Differential accumulation of flavonoids and phytohormones resulting from the canopy/rootstock interaction of citrus plants subjected to dehydration/rehydration. Plant Physiology and Biochemistry, 119: 147-158. DOI https://doi.org/10.1016/j.plaphy.2017.08.019

Schmelz, E.A., Grebenok, R.J., Ohnmeiss, T.E. \& Bowers, W.S. (2002). Interactions between Spinacia oleracea and Bradysia impatiens: A role for phytoecdysteroids. Archives of Insect Biochemistry and Physiology, 51: 204-221. DOI 10.1002/arch.10062

Slámová, K., Kapešová, J. \& Valentová, K. (2018). "Sweet Flavonoids": GlycosidaseCatalyzed Modifications. Int J Mol Sci, 19: 2126. DOI 10.3390/ijms19072126

Smilde, A.K., Westerhuis, J.A. \& De Jong, S. (2003). A framework for sequential multiblock component methods. Journal of Chemometrics, 17: 323-337. DOI 10.1002/cem.811

Stevenson, P.C., Kimmins, F.M., Grayer, R.J. \& Raveendranath, S. (1996). Schaftosides from rice phloem as feeding inhibitors and resistance factors to brown planthoppers, Nilaparvata lugens. IN Städler, E., Rowell-Rahier, M. \& Bauer, R. (Eds.) Proceedings of the 9th International Symposium on Insect-Plant Relationships. Springer Netherlands.

Sumner, L.W., Amberg, A., Barrett, D., Beale, M.H., Beger, R., Daykin, C.A., Fan, T.W.M., Fiehn, O., Goodacre, R., Griffin, J.L., Hankemeier, T., Hardy, N., Harnly, J., Higashi, R., Kopka, J., Lane, A.N., Lindon, J.C., Marriott, P., Nicholls, A.W., Reily, M.D., Thaden, J.J. \& Viant, M.R. (2007). Proposed minimum reporting standards for chemical analysis Chemical Analysis Working Group (CAWG) Metabolomics Standards Initiative (MSI). Metabolomics : Official journal of the Metabolomic Society, 3: 211-221. DOI 10.1007/s11306-007-0082-2

Tenango, M.P., Hernández, M.S. \& Hernández, E.A. (2017). Flavonoids in Agriculture, Flavonoids - From Biosynthesis to Human Health. IN Justino, G.C. (Ed.): IntechOpen. 
Toojinda, T., Tragoonrung, S., Vanavichit, A., Siangliw, J.L., Pa-In, N., Jantaboon, J., Siangliw, M. \& Fukai, S. (2005). Molecular Breeding for Rainfed Lowland Rice in the Mekong Region. Plant Production Science, 8: 330-333.

Uawisetwathana, U., Graham, S., Kamolsukyunyong, W., Sukhaket, W., Klanchui, A., Toojinda, T., Vanavichit, A., Karoonuthaisiri, N. \& Elliott, C. (2015). Quantitative 1H NMR metabolome profiling of Thai Jasmine rice (Oryza sativa) reveals primary metabolic response during brown planthopper infestation. Metabolomics, 11: 16401655. DOI 10.1007/s11306-015-0817-4

War, A.R., Paulraj, M.G., Ahmad, T., Buhroo, A.A., Hussain, B., Ignacimuthu, S. \& Sharma, H.C. (2012). Mechanisms of plant defense against insect herbivores. Plant Signaling \& Behavior, 7: 1306-1320. DOI 10.4161/psb.21663

Wishart, D.S., Jewison, T., Guo, A.C., Wilson, M., Knox, C., Liu, Y., Djoumbou, Y., Mandal, R., Aziat, F., Dong, E., Bouatra, S., Sinelnikov, I., Arndt, D., Xia, J., Liu, P., Yallou, F., Bjorndahl, T., Perez-Pineiro, R., Eisner, R., Allen, F., Neveu, V., Greiner, R. \& Scalbert, A. (2013). HMDB 3.0-The Human Metabolome Database in 2013. Nucleic Acids Research, 41: D801-D807. DOI 10.1093/nar/gks1065

Wittstock, U. \& Gershenzon, J. (2002). Constitutive plant toxins and their role in defense against herbivores and pathogens. Current Opinion in Plant Biology, 5: 300-307. DOI http://dx.doi.org/10.1016/S1369-5266(02)00264-9

Wongpornchai, S., Sriseadka, T. \& Choonvisase, S. (2002). Identification and Quantitation of the Rice Aroma Compound, 2-Acetyl-1-pyrroline, in Bread Flowers (Vallaris glabra Ktze). Journal of Agricultural and Food Chemistry, 51: 457-462. DOI $10.1021 / \mathrm{jf} 025856 \mathrm{x}$

Xu, Y., Correa, E. \& Goodacre, R. (2013). Integrating multiple analytical platforms and chemometrics for comprehensive metabolic profiling: application to meat spoilage detection. Anal Bioanal Chem, 405: 5063-5074. DOI 10.1007/s00216-013-6884-3

Xu, Y. \& Goodacre, R. (2012). Multiblock principal component analysis: an efficient tool for analyzing metabolomics data which contain two influential factors. Metabolomics, 8: 37-51. DOI 10.1007/s11306-011-0361-9

Zhang, J., Li, Y., Guo, J., Du, B., He, G., Zhang, Y., Chen, R. \& Li, J. (2018). Lipid profiles reveal different responses to brown planthopper infestation for pest susceptible and resistant rice plants. Metabolomics, 14: 120. DOI 10.1007/s11306-018-1422-0 


\section{Global metabolite profiles of rice brown planthopper-resistant traits reveal}

2 potential secondary metabolites for both constitutive and inducible

\section{3 defenses}

4

5 Umaporn Uawisetwathana $^{\mathrm{a} *}$, Olivier P. Chevallier ${ }^{\mathrm{b}},{\mathrm{Yun} \mathrm{Xu}^{\mathrm{c}} \text {, Wintai Kamolsukyeunyong }}^{\mathrm{d}}$, 6 Intawat Nookaew ${ }^{\mathrm{e}}$, Thapakorn Somboon ${ }^{\mathrm{a}}$, Theerayut Toojinda ${ }^{\mathrm{d}, \mathrm{f}}$, Apichart Vanavichit ${ }^{\mathrm{g}}$, 7 Royston Goodacre ${ }^{\mathrm{c}}$, Christopher T. Elliott ${ }^{\mathrm{b}}$, Nitsara Karoonuthaisiri ${ }^{\mathrm{a}}$

8

$9 \quad{ }^{a}$ Microarray Laboratory, National Center for Genetic Engineering and Biotechnology 10 (BIOTEC), National Science and Technology Development Agency (NSTDA), Khlong 11 Nueng, Khlong Luang, Pathumthani 12120, Thailand

$12{ }^{b}$ Institute for Global Food Security, School of Biological Sciences, Queen's University 13 Belfast, Stranmillis Road, Belfast BT9 5AG, Northern Ireland

$14{ }^{\mathrm{c}}$ Department of Biochemistry, Institute of Integrative Biology, University of Liverpool, 15 Liverpool L69 3BX, United Kingdom

$16{ }^{\mathrm{d}}$ Rice Gene Discovery and Utilization Laboratory, Innovative Plant Biotechnology and 17 Precision Agriculture Research Team, National Center for Genetic Engineering and 18 Biotechnology (BIOTEC), National Science and Technology Development Agency 19 (NSTDA), Khlong Luang, Pathum Thani, Thailand

$20{ }^{\mathrm{e}}$ College of Medicine, Department Biomedical Informatics, University of Arkansas for 21 Medical Sciences, Little Rock, AR, United States

$22{ }^{\mathrm{f}}$ Integrative Crop Biotechnology and Management Research Group, National Center for 23 Genetic Engineering and Biotechnology (BIOTEC), National Science and Technology

24 Development Agency (NSTDA), Khlong Luang, Pathum Thani, Thailand. 
$25{ }^{\mathrm{g}}$ Agronomy Department, Faculty of Agriculture at Kamphaeng Saen, Kasetsart University, 26 Kamphaeng Saen, Nakhon Pathom, Thailand.

27

$28 *$ corresponding author

29 Tel.: +66-2-5646700 ext $3265 \quad$ Fax: +66-2-5646707

30 Email: umaporn.uaw@biotec.or.th

31 Abbreviated title: Rice metabolomics analysis of brown planthopper-resistant traits 


\section{Abstract}

Brown planthopper $(\mathrm{BPH})$ is a phloem feeding insect that causes annual disease outbreaks, called hopper burn in many countries throughout Asia, resulting in severe damage to rice production. Currently, mechanistic understanding of $\mathrm{BPH}$ resistance in rice plant is limited, which has caused slow progression on developing effective rice varieties as well as effective farming practices against $\mathrm{BPH}$ infestation. To reveal rice metabolic responses during 8 days of $\mathrm{BPH}$ attack, this study examined polar metabolome extracts of $\mathrm{BPH}$ susceptible (KD) and its BPH-resistant isogenic line (IL308) rice leaves using UPLC-QToFMS combined with multi-block PCA. This multivariate statistical model revealed different metabolic response patterns between the $\mathrm{BPH}$-susceptible and $\mathrm{BPH}$-resistant varieties during BPH infestation. The metabolite responses of the resistant IL308 variety occurred on Day 1, which was significantly earlier than those of the susceptible KD variety which showed an induced response by Days 4 and 8 . BPH infestation caused metabolic perturbations in purine, phenylpropanoid, flavonoid, and terpenoid pathways. While found in both susceptible and resistant rice varieties, schaftoside (1.8 fold), iso-schaftoside (1.7 fold), rhoifolin (3.4 fold) and apigenin6-C- $\alpha$-L-arabinoside- $8-C-\beta$-L-arabinoside levels (1.6 fold) were significantly increased in the resistant variety by Day 1 post-infestation. 20-hydroxyecdysone acetate $(2.5$ fold) and dicaffeoylquinic acid (4.7 fold) levels were considerably higher in the resistant rice variety than those in the susceptible variety, both before and after infestation, suggesting that these secondary metabolites play important roles in inducible and constitutive defenses against the BPH infestation. These potential secondary metabolites will be useful as metabolite markers and/or bioactive compounds for effective and durable approaches to address the BPH problem.

Keywords: LC-HRMS; multi-block principal component analysis; Metabolite profiling; Brown planthopper resistance; Thai Jasmine rice; Oryza sativa 


\section{Introduction}

Rice is the staple food of more than half of the world's population. Thai Jasmine rice is recognized worldwide for its fine quality due to its jasmine scent and unique texture

(Wongpornchai et al., 2002). However, this rice variety is highly susceptible to insect infestation, especially brown planthopper (BPH), which causes devastating production yield losses (Heong, 2009). Pesticides have been used to control the disease, but they are toxic and harmful to humans and their residues can contaminate the crops (Heinrichs, 1979). To

overcome this problem with an environmentally-friendly and sustainable production strategy, rice breeding programs have successfully produced $\mathrm{BPH}$-resistant isogenic lines of Thai Jasmine rice using a backcross introgression technique from the donor BPH-resistant Rathu Heenati (RH) to the recipient Thai Jasmine rice (Toojinda et al., 2005, Jairin et al., 2009).

The resulting rice isogenic lines conserved the aroma and texture characteristics of Thai Jasmine rice, as well as carrying BPH resistance loci from RH rice variety.

The $\mathrm{RH}$ rice variety, a native rice from Sri Lanka, is well known as possessing a broad spectrum range of BPH resistance to four of the most dominant BPH biotypes (Fujita et al., 2013). In the post-genomic era, linkage map analysis showed that RH variety contains three main BPH resistance loci on chromosomes 4, 6 and 10 (Hu et al., 2016). Two major BPH resistant genes (Bph3 and Bph32) have been characterized by map-based cloning. Bph3 located on short arm of chromosome 4 was identified as a plasma-membrane-localized lectin receptor kinase (Liu et al., 2015), and Bph32 located on short arm of chromosome 6 was identified as an unknown short consensus repeat (SCR) domain-containing protein localized on the plasma membrane of the cells. The BPH-resistant rice varieties conferred an antibiosis resistance to BPH and Bph32 is highly expressed in the leaf sheaths (Ren et al., 2016). The introgression line carrying Bph3 and Bph32 showed an increased expression level of the OsSTPS2 (sesquiterpene synthase 2) transcript located on chromosome 4 
82 (Kamolsukyeunyong et al., 2019). Pitija et al. also reported elevated levels of monoterpenoids 83 in the BPH-resistant rice varieties (IL162, IL283, IL302 and IL308) when compared to the susceptible KD cultivar using GC-MS analysis (Pitija et al., 2014). Moreover, LOC_Os06g03514 transcript (a putative metallopeptidase), a gene involved in catalyzing reactive oxygen species, was induced in the resistant rice variety after BPH infestation (Kusumawati et al., 2018). Feeding and behavioral studies revealed that BPH preferred settling on susceptible Taichung Native 1 (TN1) to BPH-resistant IL308 variety (Kamolsukyunyong et al., 2013) and did not prefer feeding on BPH-resistant RH variety as determined by lower honeydew excretion compared to the susceptible variety (Kusumawati et al., 2018), suggesting that the BPH-resistant rice showed antixenosis and antibiosis activities. The elucidation of BPH resistance loci from the previous studies were conducted at gene structure and gene expression level. Recently, high-resolution mapping and breeding technique has been applied to identify a novel brown planthopper resistance gene derived from wild rice (Oryza. rufipogon Griff) (Li et al., 2019). However, there is still a lack of knowledge regarding the metabolic response mechanism associated with BPH infestation between susceptible and resistant rice (carrying Bph3 and Bph32) lines that need further investigation to provide better understanding of BPH resistance mechanisms.

Plants generally respond to abiotic and biotic stresses by producing different metabolites, particularly, secondary metabolites which play significant roles in plant defense mechanism against herbivores attack (Balmer et al., 2013, Nakabayashi and Saito, 2015). Previous studies employed ${ }^{1} \mathrm{H}$ NMR-based metabolomics using methanol-water extraction to identify primary metabolic response of BPH-resistant rice carrying Bph 14 (Liu et al., 2010, Liu et al., 2017) and Bph3 (Uawisetwathana et al., 2015) and revealed that metabolites involved in GABA shunt, glycolysis, amino acid biosynthesis, purine/pyrimidine biosynthesis changed in response to BPH infestation. Gas chromatography-based metabolomics using 
methanol-hexane extraction was employed to study primary and secondary metabolic responses of rice carrying Bph6 revealing sterol and phytol biosyntheses as important metabolic pathways against BPH attack (Zhang et al., 2018). In addition, a combination of liquid chromatography-based metabolomics using methanol-water extraction and gas chromatography-based metabolomics using methoxy-amino-hydrochloride and MSTFA derivatization was used to reveal changes in levels of several metabolites such as cyanoamino acids, thiamine, taurine and hypotaurine-metabolism, and quercetin and spermidine metabolites as a response in rice carrying $B p h 1, b p h 2, B p h 3$ and $B p h 32$ under BPH infestation (Kang et al., 2019). These studies suggested that metabolites contributing to particular BPH resistant genes in rice were diverse. However, metabolites, especially secondary metabolites related to the rice carrying only $B p h 3$ and $B p h 32$, have not been explicitly identified yet.

To investigate plant metabolites under biotic stress condition, ultra-high performance liquid chromatography-quadrupole time of flight-mass spectrometry (UHPLC-QToF-MS) was employed as it is a powerful tool with high resolution which provides excellent mass accuracy, as well as its wide metabolites coverage which supports the detection of both primary and secondary metabolites (Allwood and Goodacre, 2010). In combination with multivariate statistical analysis, multi-block principal component analysis (MBPCA) has been shown to accelerate data fusion and data interpretation, particularly in the experiments influenced by multiple factors (Xu and Goodacre, 2012). Successful application of MS-based metabolomics in combination with MBPCA has been used for metabolite profiling such as to study multi-platforms metabolome in melon (Biais et al., 2009), to monitor the natural spoilage process in pork (Xu et al., 2013) and to study the effects of post flowering environmental conditions on blackcurrant fruit (Allwood et al., 2019). 
In this study, UPLC-QToF-MS was employed to profile and pinpoint the key

metabolites differentially altered between the BPH-susceptible Thai Jasmine rice and its

BPH-resistant isogenic line (IL308). The comparison of the samples with and without BPH infestation for three different time points (Days 1,4 and 8) revealed that a specific group of secondary metabolites involved in phenylpropanoid, flavonoid or terpenoid metabolism plays a significant role in both constitutive and inducible defenses in the rice samples.

\section{Materials and Methods}

\section{Rice and brown planthopper (BPH) samples}

Brown planthopper (BPH, Nilaparvata lugens) biotype Ubon Rachathani (UBN-BPH)

was cultured in a standard BPH-susceptible Taichung Native 1 (TN1) rice cultivar in a closed system. Paddy soil without fertilizer was used for planting of TN1 in the pots.

Approximately, 50-100 TN1 seeds were germinated on each pot. Two to three-week-old seedlings of TN1 were used for insect cultivation. The reared $\mathrm{BPH}$ population at third to fourth-instar nymph stage was used for rice infestations (Jairin et al., 2009).

Rice samples used in this study were BPH-susceptible Thai jasmine rice KDML105 (KD) and BPH-resistant isogenic line (UBN3078-101-432-6-308: IL308). IL308 is the BPHresistant IL of KD containing Bph3, Bph 32 and OsSTPS2 (Liu et al., 2015, Ren et al., 2016, Kamolsukyeunyong et al., 2019).

\section{BPH treatment and rice sample collection}

The BPH treatment was performed as depicted in Figure S1. Paddy soil collected

from the field was used in this experiment. Rice seeds were germinated in the pots for one week. Then, water was added into each pot to cover the soil. At 3-week-old seedling stage, one gram of nitrogen fertilizer (46-0-0) was added to each pot. The water was always maintained to cover the soil during the experiment. All rice varieties examined in this study 
were grown in a total of 36 pots (18 pots/each variety; pot dimensions: $20 \mathrm{~cm}$ in diameter and $18 \mathrm{~cm}$ in height) with 3 plants per pot in a greenhouse with $13 \mathrm{~h}(06: 00-19: 00)$ light and $11 \mathrm{~h}$ (19:00 - 06:00) dark cycles for 45 days (tilling stage) before dividing the rice samples into two groups (with and without BPH treatment, 9 pots/group). For the BPH treatment group, each rice variety was transferred into an aluminum cage covered with plastic mesh $(1 \mathrm{~mm}$ diameter) and second- to third-instar BPH nymphs were then introduced to each plant (100$150 \mathrm{BPH}$ nymphs/pot). In parallel, the groups without BPH treatment were maintained in separate cages as control groups.

Three biological replicates (three separate pots) of the rice leaf samples in each variety were collected at three different time points (Days 1,4, and 8). The samples including leaf sheathes and leaf blades were cut on the lowest point of the leaf sheath (approximately 60-100 cm tall) before quickly freezing in liquid nitrogen. All frozen samples were lyophilized (Modulyo®, Thermo Scientific, USA), ground to a fine powder (Phillip HR2020, USA) and stored at $-80^{\circ} \mathrm{C}$ prior to analysis.

\section{Metabolite extraction}

An in-house extraction protocol was used in this study with some modifications 172 (Carrizo et al., 2017, O’Kane et al., 2013). Đried-Freeze-dried leaf powder (100 mg) was 173 extracted with $2 \mathrm{~mL}$ of 3:2(v/v) methanol:water solution by mixing for $10 \mathrm{~min}$ at room 174 temperature using a minimix standard shaker (Merris Enginering, U.K.) before sonicating for $17515 \mathrm{~min}$ at $4^{\circ} \mathrm{C}$ in a ultrasonic bath sonicator. Extracts were centrifuged at $3,500 \times g$ at $4^{\circ} \mathrm{C}$ for $17620 \mathrm{~min}$ (Sorvall Legend, Thermo Scientific). Supernatant was collected for polar metabolome using $0.22 \mu \mathrm{m}$ Costar Spin-X® Centrifuge Tube Filter $\left(10,000 \times g\right.$ at $4^{\circ} \mathrm{C}$ for $\left.5 \mathrm{~min}\right)$. The 
181 prior to ultra-high pressure liquid chromatography-quadrupole time of flight-mass

182 spectrometry (UPLC-QToF-MS) analysis.

\section{Untargeted metabolite profiles using LC-QToF-MS}

Samples-Filtered extracts were analysed using an Acquity UPLC I-class system (Waters, MA, USA) which coupled an Acquity UPLC® HSS T3 (2.1 x 100 mm, $1.8 \mu \mathrm{m})$ column (Waters, USA) with a Xevo G2S QToF mass spectrometer (Waters, MA, USA). The flow rate was $0.4 \mathrm{~mL} / \mathrm{min}$ and the column temperature was maintained at $45^{\circ} \mathrm{C}$. Mobile phases were water with $0.1 \%$ formic acid (A) and methanol with $0.1 \%$ formic acid (B). Sample manager temperature was set at $6^{\circ} \mathrm{C}$ and an aliquot of $5 \mu \mathrm{L}$ was injected onto the column using the following method: an isocratic period for 0.50 min at 99:1 (A:B) followed by a linear gradient to $1: 99(\mathrm{~A}: \mathrm{B})$ over $16.50 \mathrm{~min}$ which was maintained for $1.75 \mathrm{~min}$ before restoring to an initial condition $(99: 1, \mathrm{~A}: \mathrm{B})$ for $1.25 \mathrm{~min}$. For reproducibility measurement of the instrument, pooled biological samples were used as quality control samples injected every 10 samples (Dunn et al., 2011, Broadhurst et al., 2018). Prior to the experiment, system suitability (LC and MS) was assessed by 3 consecutive injections of commercially available reference standard (Waters "test mix", part 186006963). A lock-mass solution of Leucine Enkephalin $(1 \mathrm{ng} / \mu \mathrm{L})$ in acetonitrile/water containing $0.1 \%$ formic acid $(1: 1, \mathrm{v} / \mathrm{v})$ was continuously infused into the MS via the lock-spray at a flow rate of $10 \mu \mathrm{L} / \mathrm{min}$. Each filtered extract was randomly injected three times to obtain three technical replicates throughout the experiment $(n=3 \times 3)$. From the nine values, the mean was calculated. The means from all groups were compared and statistically analyzed as described in details in data fusion and data analysis section.

The MS data were acquired in both positive and negative electrospray ionization (ESI) modes over the range 50-1200 Da. The ionization source parameters in positive mode were as follows: capillary voltage $1.0 \mathrm{kV}$, sample cone voltage $30 \mathrm{~V}$, source temperature 
$120^{\circ} \mathrm{C}$, desolvation temperature $450^{\circ} \mathrm{C}$, desolvation gas flow $850 \mathrm{~L} / \mathrm{h}$ and cone gas flow 25

$\mathrm{L} / \mathrm{h}$. For negative mode, the following settings were applied: capillary voltage $2.0 \mathrm{kV}$, sample cone $30 \mathrm{~V}$, source temperature $120^{\circ} \mathrm{C}$, desolvation temperature $450^{\circ} \mathrm{C}$, desolvation gas flow $850 \mathrm{~L} / \mathrm{h}$ and cone gas flow $25 \mathrm{~L} / \mathrm{h}$. Mass spectra data were acquired in continuum mode with a scan time of $0.1 \mathrm{sec}$ using MSE function (low energy: $4 \mathrm{eV}$, high energy: ramp from 10 to $30 \mathrm{eV}$ in both positive and negative modes).

Spectra from the LC-MS analysis were analysed using MassLynx version 4.1 and Progenesis QI softwares (Waters, USA). Briefly, raw chromatogram data acquired by MassLynx software were transformed into 2D matrices between retention time and $\mathrm{m} / \mathrm{z}$. The data were preprocessed using Progenesis QI software by alignment and peak picking. For peak picking, a minimum peak width filter of 0.06 min was applied. Progenesis QI uses ratiometric data in log space, along with a median and mean absolute deviation outlier filtering approach, to calculate the scalar factor for normalization and is able to determine which pairs (or groups) of compound ions might be different adduct forms of the same compound. For 2 compound ions to be considered part of the same compound they must have the same retention time and ion masses that differ by an amount equal to the mass difference between 2 experiments. These programs quantify and aid identification of the integrals from all peaks above the threshold deemed to be of interest.

\section{Data fusion and data analyses}

Principal component analysis was applied to auto-scaled data matrices for first visualization and assess the stability of the analytical platform. Multi-block principal component analysis (MBPCA) was then employed for two purposes: (1) to fuse both positive and negative mode data together, and (2) visualize the pattern of the data as a whole (Xu et al., 2013). The data collected from both LC-MS positive and negative modes were placed side-by-side and matched on the sample level (i.e. each row is the same sample analysed on 
positive and negative mode respectively). Each block was firstly auto-scaled so that the mean and standard deviation of each variable were zero and 1, respectively. Block scaling (i.e. the data within each block were divided by the square root of the number of variables in that block) was then applied to compensate for the variance differences caused by different number of variables in different modes. Consensus principal component analysis (CPCA), a commonly used MBPCA model (Smilde et al., 2003), was then applied to gain a consensus view of the pattern of the data collected from both ionization modes.

Another type of analysis is to decouple the effects from multiple influential factors (Xu and Goodacre, 2012). In this study, there are three main factors: temporal factor, genotypes, and treatment of BPH. To be able to view the effect of one factor without interference from the other factors, CPCA was applied to both ESI positive and negative ion modes separately. On each mode of data, the samples were partitioned to three blocks according to time, each block has all the samples on the same time point while between different blocks the samples were matched on both genotype and treatment; i.e., on each row the samples on the three blocks have the same genotype and BPH treatment (either treated or control). Auto-scaling was applied to each data block prior to CPCA.

Screening workflow was shown in Figure 1. In the first screening, three-way analysis of variance (3-way ANOVA) with false discovery rate $<0.001$ and $p$-value $<0.05$ has been employed to identify metabolites which varied significantly at least genotype factor, interactions by genotype/treatment, genotype/time and genotype/treatment/time. In the second screening, the selected metabolite features were transformed to log value and auto scaled prior to hierarchical clustering based on metabolite patterns with their level changes $>1.5$ folds using Cluster 3.0 software (Eisen et al., 1998). In the third screening, the selected metabolite features were tested for significant differences between control and treatment groups per time per genotype using Mann-Whitney U test $\left(^{*} p<0.05, * * p<0.01\right.$ and $* * *$ 
$256 p<0.001)$. The response of each sample ( $\mathrm{n}=3 \times 3$ /group) was calculated as the mean values

257 with standard deviation (SD). Multi-block analysis and three-way ANOVA were performed 258 in MATLAB 2016a (Mathworks, MA, U.S.A). Mann-Whitney U test was performed in 259 GraphPad Prism 5.01 (GraphPad Software, San Diego, CA). The metadata was available at 260 the MetaboLights metabolomics data repository with an identifier MTBLS1197 (URL 261 www.ebi.ac.uk/metabolights/MTBLS1197).

\section{Metabolite identification}

Metabolite features were identified according to the minimum reporting standards for chemical analysis defined by the Metabolomics Standards Initiative (MSI) (Sumner et al., 2007). The ions with identified adduct forms and neutral masses were automatically matched to their MS spectra and monoisotopic masses using external public databases including ChemSpider (http://www.chemspider.com/), HMDB (Wishart et al., 2013), BioCyc (http://biocyc.org/), Plant Metabolic Network (http://www.plantcyc.org/), NIST (http://www.nist.gov/), LipidMAPS (http://www.lipidmaps.org/) and KEGG (http://www.genome.jp/kegg/) provided in Progenesis software. The parameter criteria were composed of precursor tolerance at $10 \mathrm{ppm}$, fragment tolerance at $10 \mathrm{ppm}$ and isotope similarity filter at $95 \%$. Additionally, the selected ions that could not be identified from aforementioned databases were manually matched to their monoisotopic masses using Metlin (https://metlin.scripps.edu). Precursor tolerance was set at $30 \mathrm{ppm}$. For MSI level 1, the identities of metabolite features were confirmed by using authentic standards (isoschaftoside, schaftoside, dicaffeoylquinic acid; Sigma, USA). For MSI level > 1, the identities of these metabolite features were annotated based on MS spectra (precursor ions and their pseudo MS/MS acquired by $\mathrm{MS}^{\mathrm{E}}$ function of QToF-MS) using the public databases above. 


\section{Results}

\section{Phenotypic response affected by BPH infestation}

The temporal effect of BPH infestation on rice leaf between susceptible and resistant rice varieties was investigated. First, leaves from both rice varieties were visibly the same without $\mathrm{BPH}$ infestation, while those in the $\mathrm{BPH}$-treated groups were clearly different on Day 4 and more obvious by Day 8 (Figure 2). Specifically, the leaf samples in the BPHsusceptible KD cultivar (KD) turned yellow and dried, exhibiting the classical hopper burn symptoms, whereas those of the BPH-resistant IL308 variety (IL308) were not affected by the infestation. Liquid chromatography-high resolution mass spectrometry (UPLC-QToFMS) was then employed to determine metabolic response associated with the BPH infestation.

\section{Global metabolic response affected by BPH infestation}

Base peak intensity (BPI) chromatograms of the samples in this study showed differences across the rice varieties, along the time points and between them with and without $\mathrm{BPH}$ treatment in both positive electrospray ionization (ESI+) and negative electrospray ionization (ESI-) modes. Representative BPI chromatograms of BPIs of the leaf extracts are shown in Figure $\mathbf{S 2}$.

Following peak peaking, the data were normalized within the Progenesis software (4,688 ions for ESI+ mode and 2,151 ions for ESI- mode) as detailed in the Materials and Methods, and were then subjected to PCA and MBPCA for visualization of global metabolic profiles among the sample groups. The scores plot of PCA was given in Figure $\mathbf{S 3}$ and it showed that all the QC samples were tightly clustered together, indicating that the analytical platform was stable during the course of analysis. Unlike classical PCA, MBPCA models such as CPCA produce two sets of scores, namely super scores and block scores. The super 
scores showed "common trend" across different blocks, while block scores showed unique patterns on each block. The MBPCA super scores plot revealed distinctive differences among the time points of the experiment, and showed slight differences on the BPH treatment with 44.4\% total explained variance (TEV) in PC1 and 13.5\% TEV in PC2. However, these analyses did not show obvious differences between the rice varieties (Figure S4). This result suggested that the temporal effect of the experiment was a major influential factor for the separation between the metabolic profiles which corresponding to the occurrence of the hopper burn. Consequently, further time block scores plots were performed to remove the temporal factor in the dataset in order to determine the effect of rice genotype and BPH treatment between susceptible and resistant rice varieties during the BPH infestation (Figure 3A-B).

\section{Data obtained from both ESI+ and ESI- showed similar patterns of metabolic} adaptations as illustrated in Figure 3. Day 1 block scores plot (ESI+ : PC1=14.8\% TEV and PC2=11.7\% TEV; ESI- : PC1=12.6\% TEV and PC2=11.3\% TEV) showed slightly different profiles between susceptible KD and resistant IL308 varieties after the BPH infestation, whereas Day 4 block (ESI+ : PC1=61.0\% TEV and PC2=8.7\% TEV; ESI- : PC1=50.5\% TEV and PC2 $=10.7 \%$ TEV) and Day 8 block (ESI+ : PC1=27.0\% TEV and PC2 $=26.6 \%$ TEV, ESI- : PC1=50.5\% TEV and PC2=10.7\% TEV) scores plots showed more dramatic differences between the susceptible KD and the resistant IL308 varieties within the BPHinfested groups. The results clearly revealed different metabolic responses to the BPH infestation between the susceptible and the resistant varieties. By contrast, the profiles in the control groups were similar in all the time blocks, indicating similar metabolic states of the two groups.

To identify key metabolites with significant changes, the processed data were subsequently analyzed according to the data analysis workflow and summarized results 
shown in Figure 1. Three-way ANOVA indicated that a total of 801 features in ESI+ and 444 features in ESI- significantly contributing to these differences; i.e., metabolite features with adjusted $p$-value $<0.05$ after FDR $<0.001$ and $p$-value $<0.05$-at least genotype factor, two factors by genotype/treatment and genotype/time, and multiple factors by genotype/treatment/time were selected to be significantly different (Table S1). Of these, cluster analysis revealed that a total of 40 metabolite features in ESI+ and 56 metabolite features in ESI- could be grouped into five different alteration patterns, followed by MannWhitney U test for control versus BPH-infested groups per genotype per time. This allows elucidation of significant metabolites associated with the BPH infestation (Table S2).

Subsequently, the significant metabolite features (a total of 9 features from ESI+ and 18 features from ESI-) were tentatively identified (Table S3).

\section{Alteration patterns and significant metabolites affected by BPH infestation}

Different patterns were observed in the clustergram in both profiles obtained from ESI+ (Figure 4A) and from ESI- (Figure 4B), providing diverse range of metabolite features that were changes in the plant host in response to the BPH infestation. While there was a common response found in both rice varieties, there were also different responses between the susceptible and the resistant rice, particularly with different response times after the infestation. The susceptible rice showed induced response at the latter time points (Days 4 and 8), whereas the resistant rice showed both constitutive and induced responses at the early timepoint (Day 1) and at the late timepoint (Day 8) (Figure 4A-B). Of these, three major responses were observed in Figures $4 \mathrm{~A}$ and $\mathbf{4 B}$ and these alteration patterns are described below:

First, the common stress response (Cluster I of both ESI modes: ESI+ (9 features) and ESI- (14 features)) represented the same alteration pattern between the susceptible and 
the resistant rice varieties. The metabolite levels in this cluster was increased on Day 1 in both control and treatment groups, but only remained at this increased level until Day 4 only in the treatment groups. Among these metabolite features, four $\left(3.44 \_477.1210 \mathrm{~m} / \mathrm{z}\right.$, 8.98_688.2016n, 8.98_657.1760m/z and 9.17_717.1974m/z) were found to be significantly changed. Given their common pattern in both rice varieties, they might be involved in a core metabolic activity essentially for growth and/or involved in a stress response which might not directly be associated with the resistance process.

Second, the unique response in the susceptible KD variety (Cluster IIa, IIb and IIc of ESI+: 7, 6 and 13 features, respectively and Cluster II of ESI-: 14 features) represented metabolites whose levels were increased only in the susceptible variety at late stage (Days 4 and 8) of the infestation. Nine of these metabolite features $\left(1.34 \_169.0361 \mathrm{~m} / \mathrm{z}\right.$, 2.47_137.0478m/z, 3.29_228.0502m/z, 4.31_277.1655m/z, 5.47_243.1346m/z, 5.95_272.1731n, 6.09_274.1205m/z, 9.24_571.1976m/z and 9.25_537.2135m/z) were found to be significantly changed. Of the nine features, three metabolites were identified as uric acid (Level 2 of MSI, 4 and 2.2 fold increase), hypoxanthine (Level 1 of MSI, 6 and 5.6 fold increase) and 4-(L-Alanin-3-yl)-2-hydroxy-cis,cis-muconate 6-semialdehyde (Level 2 of MSI, 1.5 and 2.7 fold increase) (Table S3), which belong to purine and amino acid metabolism. These late induction patterns after the infestation suggested that these metabolites might not be effective biomolecules for defense against the insect. However, the alteration patterns might be related to the hopper burn symptom in the susceptible variety, suggesting that these metabolite changes may be associated with cell death or the biotic stress response. These metabolites might be used as markers of the progression of hopper burn.

Third, the unique response in the resistant IL308 rice variety (Cluster III of ESI+ and ESI-) represented three different metabolite alteration patterns whose levels were significantly higher than those in the susceptible variety as in the following subgroups: 
The first subgroup, constitutive response (Cluster IIIa of ESI-: 9 features) represented metabolites whose levels were significantly higher than those in the susceptible variety in both before and after the infestation. Three significantly changed metabolite features $\left(9.73 \_515.1185 \mathrm{~m} / \mathrm{z}, 7.97 \_577.1516 \mathrm{~m} / \mathrm{z}\right.$ and $\left.11.38 \_583.3074 \mathrm{~m} / \mathrm{z}\right)$ were identified as dicaffeoylquinic acid (Level 1 of MSI, 4.7 fold increase), and 20-hydroxyecdysone acetate (Level 2 of MSI, 2.5 fold increase) which belong to phenolic acid, flavonoid, and terpenoid compound classes, respectively. Given their constitutive and unique patterns in resistant variety, these metabolites might be important as constitutive specialized (secondary) metabolites to protect against the insect attack.

The second subgroup, early induction at Day1 post-infestation (Cluster III of ESI+: 5 features and Cluster IIIb of ESI-: 12 features) represented metabolites whose levels were significantly increased only in the resistant variety at Day1 post-infestation. Five metabolites (7.25_564.1431n, 7.40_564.1431n, 7.50_757.1970m/z, 7.80_533.1254m/z, 7.97_577.1516m/z and 8.21_565.1520m/z) were found to be significant different, and identified (Level 1 or 2; see Table S3) as iso-schaftoside (1.7 fold increase), schaftoside (1.8 fold increase), rhoifolin (Level 2 of MSI, 3.4 fold increase), apigenin-6-C-glucoside-8-Carabinoside, cyanidin 3-O-(6-O-p-coumaroyl) glucoside-5-O-glucoside, apigenin 6-C- $\alpha$-Larabinoside- 8 -C- $\beta$-L-arabinoside and naringenin 8 -C- $\alpha$-L-arabinopyranosyl-7-O- $\beta$-Dglucopyranoside belonging to flavonoid glycosides. These metabolites in this alteration pattern might be involved in inducible defense against the insect attack.

The third subgroup, late induction at Day8 post-infestation (Cluster IIIc of ESI-: 7 features) represented metabolites whose levels increased only in the resistant variety at Day8 postinfestation. Three significant metabolite features (8.36_245.1383m/z, 12.36_241.1430m/z and 12.52_227.1638m/z) were tentatively identified as 3-hydroxydodecanedioic acid, 2,4- 
dioxotridecanoic acid and 12-oxotridecanoic acid (all Level 2 of MSI) belonging to fatty acid class. However, there were no scientific evidences about these fatty acids in plants and other organisms. Based on the increasing levels of these metabolites at late stage of the infestation, they might not directly be associated with the resistance processes.

To evaluate the identified metabolites further, metabolite levels for each of the nine significant metabolites were considered (Figure 5). First, three metabolites with significant difference $(p<0.05)$ in the susceptible rice variety (uric acid, hypoxanthine and 4-(alanine-3yl)-2-hydroxy-muconate semialdehyde) were found to be similar in the control groups in both rice varieties, whereas those in the BPH-treated groups were different between KD and IL308 (Figure 5A). Specifically, uric acid levels in the KD were significantly increased by Day1 to Day 8 after the BPH treatment whereas those in the IL308 were increased by Day 4 to Day 8 which was slower response than in the KD. Hypoxanthine levels in the KD and the IL308 were significantly increased by Day 1 to Day 4 after the treatment; however, by Day 8, the hypoxanthine level was still significantly increased in the KD whereas those in the IL308 was significantly decreased $(p<0.05)$. The levels of 4-(alanine-3-yl)-2-hydroxy-muconate semialdehyde were significantly increased in the KD from Day 4 to Day 8 after the infestation whereas those in the \#308-IL308 were not changes.‥ Hypoxanthine and uric acid are involved in purine metabolism, and 4-(alanine-3-yl)-2-hydroxy-muconate semialdehyde is involved in tyrosine metabolism, suggesting that the KD variety employed primary metabolism important for its growth in response to the biotic stress.

By contrast, schaftoside, isoschaftoside, rhoifolin and apigenin- $6-\alpha-$ arabinoside- 8 $\beta$-arabinoside metabolites whose levels were significantly increased $(p<0.05)$ by $1.8,1.7,3.4$ and 1.6 fold increase, respectively, only in the resistant IL308 variety by Day 1 postinfestation, butdecreased in susceptible variety (Figure 5B). Flavonoid glycosides are part of 
the flavonoid family which has previously been reported to play role on insect resistance in several crops such as sorghum, sunflower, eucalyptus (Tenango et al., 2017), wheat (Dreyer and Jones, 1981) and maize (Mierziak et al., 2014). Therefore, these metabolites may also be involved in $\mathrm{BPH}$ resistance in rice.

Interestingly, 3,4-di-O-caffeoylquinic acid_and 20-hydroxyecdysone acetate metabolites levels were constitutively higher tevels-(4.7 and 2.5 fold increase, respectively) than those in the susceptible variety throughout the experiment (Figure 5C). After the BPH infestation, the three metabolites had different patterns. Particularly, dicaffeoylquinic acid was significantly increased $(p<0.05)$ by BPH at Day 4 and decreased at Day 8 as compared to control treatment, whereas 20-hydroxyecdysone acetate was significantly increased $(p<0.05)$ at Day 1 and decreased at Day 8. These specialized metabolites are involved in phenylpropanoid, flavonoid or terpenoid metabolism that may play important roles on BPH resistance.

\section{Discussion}

\section{Different phenotypic responses between susceptible and resistance rice varieties upon}

\section{BPH treatment}

The phenotypic response indicated that, at Day 8 after BPH infestation, the susceptible KD showed hopper burn symptom, whereas the resistant IL308 did not exhibit these symptoms and was able to continue to grow. These results agreed with previous phenotypic screening studies to evaluate BPH damage score (Jairin et al., 2009), confirming the expected BPH-resistant characteristic of the IL308 rice isogenic line of KD carrying Bph3, Bph32 and OsSTPS2 used in this study.

To decipher potential mechanism for BPH resistance, a metabolomics comparison between the resistance and susceptible rice varieties subjected to $\mathrm{BPH}$ infestation was 
undertaken. After chemometric analyses (viz. MBPCA and univariate statistical testing) significant metabolites and metabolic pathways were revealed that were different between susceptible and resistance varieties during BPH infestation, which has provided key information for metabolic adaptation of plant as defense against BPH attack (Figure 6).

\section{Metabolic response of the susceptible rice during the BPH infestation}

Hypoxanthine and uric acid levels were found in the infested susceptible rice variety in higher levels than in the control and the resistant variety. These results agree with our previous study using ${ }^{1} \mathrm{H}$-NMR-based metabolomics comparing metabolomics responses in susceptible rice variety (KD) between BPH treatment and control (no BPH treatment) at Day 1,4 and 8 and revealing that hypoxanthine level was affected in the BPH-treated group at the late time points (Day 4 and 8) (Uawisetwathana et al., 2015). These metabolites are found to be involved in purine metabolism via a salvage pathway (Ashihara et al., 2018). In the rice plant, hypoxanthine is reversibly synthesized by inosine to produce xanthine and consequently convert to uric acid. The purine salvage pathway in plants is generally employed for remobilization of nitrogen and nutrient recycling which saves energy usage (Brychkova et al., 2008). Uric acid is then finally catabolized to ammonia. The accumulation of ammonia causes cytotoxicity in plants (Hauck et al., 2014). Hypoxanthine is a prime metabolite contributing to responses to environmental and biotic stresses (Pastor et al., 2014). From this study and literature, it could be hypothesized that BPH-susceptible rice adapts itself by utilizing hypoxanthine and uric acid through salvage pathway of purine biosynthesis for its survival.

In summary, it is proposed that the susceptible rice responded to the insect attack by increased turnover of nucleotide synthesis via a salvage pathway to acclimate and maintain cellular function close to normal conditions. However, in this study, there were no secondary 
metabolites that were significantly correlating to BPH-defense processes in this variety. It could be suggested that, apart from adaptation process, there is lack of systemic defense processes in the susceptible rice.

\section{Metabolic response of the resistant rice during the BPH infestation}

Interestingly, this study also found flavonoid glycoside (MSI level 1), chlorogenic acid (MSI level 1) and hydroxytriterpenoid (MSI level 2) in the resistant rice variety. With

hydrophilic as their functional groups, these compounds are water soluble as reported in these

studies (Matsui et al., 2004, Slámová et al., 2018)_which could be extracted by

methanol/water used in this study. Flavonoid glycosides are major plant secondary

metabolites composed of glycosylated flavones, flavonols, anthocyanins, flavanones, isoflavones and etcetera which play essential roles in plants under stress conditions such as UVB screening, pollination, seed development, allellopathy and plant-pathogen interaction (Harborne and Williams, 2000, Falcone Ferreyra et al., 2012). Flavonoid glycosides have aare diverse across plant species. Thus, it is necessary to know flavonoid glycosides specific to rice, particularly in response to the $\mathrm{BPH}$ attack. In this study, several flavonoid glycosides were found to be significantly elevated in the post-infested resistant rice and these included schaftoside (apigenin 6-C- $\beta$-D-glucopyranosyl-8-C- $\alpha$-L-arabinopyranoside), iso-schaftoside (apigenin 6-C- $\alpha$-L-arabinopyranoside-8-C- $\beta$-D-glucopyranoside), rhoifolin (apigenin 7-Oneohesperidoside) and apigenin 6-C- $\alpha$-L -arabinopyranosyl-8-C- $\beta$-L-arabinopyranoside. Previous reports showed that schaftoside has been found in rice phloem sap as a BPH sucking deterrent in the Rathu Heenati cultivar (Stevenson et al., 1996) and IR 62 variety (Grayer et al., 1994). The mechanism of action of schaftoside was reported to inhibit $C D K 1$ of the BPH, causing the insect cell death (Hao et al., 2018), and has been shown to have nematocidal activity against a plant parasite Meloidogyne incogtina in the tuber species Arisaema erubescens (Du et al., 2011). Iso-schaftoside has been reported to have an inhibitory effect in 
501

502

a tick clover (Desmodium spp.) to a witchweed (Striga spp.) interaction (Hamilton et al.,

2012). There is currently no disease resistance evidence for apigenin $6-\mathrm{C}-\alpha-\mathrm{L}-$

arabinopyranosyl-8-C- $\beta$-L-arabinopyranoside. Rhoifolin, apigenin 7-O-neohesperidoside, is

a member of apigenin glycosides found in various plants such as bitter orange, bergamot, lemon, tomatoes, artichoke, banana, grape and grapefruit (Refaat et al., 2015, Gattuso et al., 2006, Gattuso et al., 2007). It has been reported to provide antioxidant, anti-inflammatory, antimicrobial and anticancer benefits to human's health (Refaat et al., 2015). In citrus plants, rhoifolin accumulation has been found to be associated with drought-tolerant condition (Santos et al., 2017). Thus, the present study revealed that the flavonoid aglycones, apigenin, was found to be major in rice leave during $\mathrm{BPH}$ infestation and the early induction of apigenin glycosides after the insect attack compared to the susceptible KD variety suggests that these metabolites may play an important role in inducible defense mechanism.

Interestingly, another response found in the resistant IL308 variety was constitutive elevation of several metabolites compared to the susceptible KD variety. The metabolites in this response contained dicaffeoylquinic acid and 20-hydroxyecdysone acetate. With regards to the plant defense against herbivores, constitutive defenses such as thorn, trichrome and chemical toxins indicate a promising plant defense mechanism as these chemicals are produced at all developmental stages and have durable broad spectrum of resistance (Kessler and Baldwin, 2002, Wittstock and Gershenzon, 2002, War et al., 2012). This will be expanded as follows:

Firstly, 3,4-di- $O$-caffeoylquinic acid is one of secondary metabolites from phenylpropanoid pathway derived by $p$-coumaric acid in combination with quinic acid. Its product is a chlorogenic acid, called caffeoylquinic acid which is a precursor to produce dicaffeoylquinic acid (Moglia et al., 2014, Clifford et al., 2017). It has been reported to have allelopathic effect to inhibit fungal (Fusarium. solani) growth in sweet potato (Harrison et al., 
2008), to have a protective effect against oxidative damage from salinity in tomatoes

(Martinez et al., 2016), as well as ultraviolet radiation protection in artichoke leaf (Moglia et al., 2008), has particularly protective effect against predators in tomatoes such as fruitworm Heliothis zea and beet armyworm Spodoptera exigua (Clifford et al., 2017, Felton et al., 1989, Kundu and Vadassery, 2019), and synthetic dicinnamoyl 4-deoxy quinic acid and its methyl ester derivatives were shown to have toxicity effect against pea aphid Acrythosiphon 532 pisum (Li et al., 2016). The mode of action has been proposed that quinones derived from 533 dicaffeoylquinic acid can react with nucleophiles and link covalently to insect protein; 534 subsequently limit the growth of herbivores (Kuijpers et al., 2012, Pierpoint, 1969, Felton and 535 Duffey, 1990). Caffeoylquinic acid is a common polyphenolic compound found in rice; 536 however, this is the first report where a 3,4-di- $O$-caffeoylquinic acid has been detected in rice 537 with potential insect resistance. Hence, in this study, a higher amount of 3,4-di- $O$ 538 caffeoylquinic acid in the resistant IL308 rice variety, than in the susceptible KD, might 539 suggest its protective function against the BPH attack.

540 Secondly, 20-hydroxyecdysone acetate is a phytoecdysteroid synthesized by plants for 541 defense against phytophagous insects (Adler and Grebenok, 1999, Dinan, 2001). The 542 chemical structures are an analogue to the insect molting hormone, ecdysteroids, which is 543 essential during the molting process of the insect, thus these compounds have potential role 544 on inhibiting insect growth (Abubakirov, 1980). 20-hydroxyecdysone is synthesized from 545 mevalonic acid in the mevalonate pathway of the plants cell using acetyl-CoA as a precursor. 546 Under insect herbivores damage and wound induction, 20-hydroxyecdysone was found to be 547 accumulated (Schmelz et al., 2002). 20-hydroxyecdysone showed antitumor, antibacterial and 548 antioxidant from Silene plants (Baltayev, 1998, Csábi et al., 2016) as well as insect growth 549 regulator in fern, purposing to be replacing synthetic insecticides for insect pest management 550 programs (Chaubey, 2018). From its linkage to inhibit insect growth, we hypothesize that 


\section{Concluding Remarks} early stage of infestation (Day1).

20-hydroxyecdysone acetate might involve as protective metabolite in rice after the BPH infestation. Not only was 20-hydroxyecdysone acetate constitutively increased in the resistant IL308 compared to the susceptible KD variety, but it was also induced by the BPH at the

In combination these results may suggest that the resistant rice variety has orchestrated several defense mechanisms using a series of metabolites related in phenylpropanoid, flavonoid or terpenoid pathways for both constitutive and inducible defenses. Furthermore, the adaptation process found in the resistant variety in the early stage of infestation resulted in the induction of increased hypoxanthine level on Day 1.

This study demonstrated the applicability of UPLC-QToF-MS combined with MBPCA to identify metabolites that were differential between BPH-susceptible KD and its BPH-resistant IL308 isogenic line, upon normal (pest free) and BPH infestation conditions.

Different metabolic responses were discovered with significant metabolites contributing to the responses between the susceptible and the resistant rice varieties being highlighted and these differential metabolites were mapped onto plant metabolite pathways. Focusing on the resistant variety, the significantly altered metabolites post-infestation were involved in phenylpropanoids, flavonoids and terpenoids metabolism. These secondary metabolites may potentially act as constitutive and inducible defenses against the $\mathrm{BPH}$ attack. The constitutive metabolites could be used as metabolite markers for the isogenic line selection without brown planthopper induction to accelerate precision breeding program. Furthermore, these bioactive metabolites could be applied by spraying onto susceptible rice plants to protect against BPH attack. In conclusion, this study explored potential specialized metabolites to defend against 
575 the insect herbivore, brown planthopper, and can be useful for plant breeding program to

576 select insect resistant plants without induction of elicitor.

\section{Acknowledgments}

579 This work was financially supported by Platform Technology Program (P-12-01893, 580 P-16-50339 and P-18-50973), National Center for Genetic Engineering and Biotechnology 581 (BIOTEC, Thailand). The PhD scholarship to Umaporn Uawisetwathana was awarded from 582 Graduate and Professional Development Division, National Science and Technology 583 Development Agency (NSTDA, Thailand).

584 Author Contribution Statement

585 NK, TT, AV, CE and RG conceived and designed research. WK and UU conducted 586 experiments. UU, OPC contributed analytical tools. UU and YX analyzed data. UU, NK, IN, 587 RG, YX, OPC, CE and TP wrote the manuscript. All authors read and approved the 588 manuscript.

\section{Competing interests}

590 The authors declare that they have no competing interests.

591 Compliance with ethical requirement

592 This article does not contain any studies with human subjects.

\section{Figure Legends}

594 Figure 1. $\underline{\text { MS }}$ D $\underline{\text { data }}$ processing and analysis workflow in both ESI+ and ESI- modes. 
Figure 2. Physiological response between control (-BPH) and treatment (+BPH) by Days 1, 4 and 8 for susceptible KD and resistant IL308 rice varieties (n=3/group). Red arrow indicates hopper burn symptom in the BPH-susceptible KD variety.

Figure 3. MBPCA time block scores plots between control (-BPH) and treatment $(+\mathrm{BPH})$ of susceptible KD and resistant IL308 rice varieties. A) Day 1, 4 and 8 scores plots obtained from ESI+ (4,688 variables) and B) Day 1, 4 and 8 scores plots obtained from ESI- $(2,151$ variables).

Figure 4. Clustergram analysis of susceptible KD and resistant IL308 rice varieties between control (-BPH) and treatment $(+\mathrm{BPH})$ by Days 1, 4 and 8 . A) Clustergrams obtained from 801 metabolite features of ESI+ showing five different alteration patterns (40 metabolite features) with their level changes greater than 1.5 folds and B) Clustergrams obtained from 444 metabolite features of ESI- showing five different alteration patterns (56 metabolite features) with their level changes greater than 1.5 folds.

Figure 5. Significant metabolites affected by $\mathrm{BPH}$ infestation ( $\mathrm{n}=3$ /group). A) three metabolites showing late induction in the susceptible KD variety, B) four metabolites showing early induction in the resistant IL308 variety and C) two metabolites showing constitutive in the resistant IL308 variety. Mann-Whitney U test was used to compare the control (solid line) and the treatment (dashed line) groups $\left({ }^{*} p\right.$-value $<0.05,{ }^{*} p$-value $<0.01$ and $* * * p$-value $<0.001)$ per time per genotype.

Figure 6. Proposed metabolic pathways affected in BPH-susceptible and BPH-resistant rice varieties in response to $\mathrm{BPH}$ infestation

\section{Supplementary data}

Supplementary Figure 1. Experimental design in this study. Paddy soil was dug from the field and put in the pots. Rice seeds were germinated in the pots for one week before water was added into the pot to cover the soil. Ten gram of nitrogen fertilizer (46-0-0) was added to 
each pot at the 3-week-old seedling stage. The water was always maintained to cover the soil during the experiment.

Supplementary Figure 2. Base peak intensity (BPI) chromatogram of leaf extracts from BPH-susceptible KD (A) and BPH-resistant IL308 (B) at Day 1 by UPLC-QToF-MS analysis in $\mathrm{ESI}+$ and ESI-. $-\mathrm{BPH}=$ control group and $+\mathrm{BPH}=$ treatment group.

Supplementary Figure 3. Principal component analysis (PCA) scores plots of rice BPHresistant traits including with quality control samples to show reproducibility. A) PCA scores plot obtained from ESI+ and B) PCA scores plot obtained from ESI-.

Supplementary Figure 4. MBPCA Super scores of rice BPH-resistant traits. Diamond symbol represents BPH-susceptible KD variety and circle symbol represents BPH-resistant IL308 variety. Clear symbol represents the samples in control group and solid symbol represents the samples in treatment group. Blue, green and red colors represent days 1,4 and 8 , respectively.

Supplementary Table 1. Significant metabolite features selected by three-way ANOVA ( $p$ value $<0.05)$ based on genotype factor and its interactions (time and treatment). A sheet "Supplementary Table 1_Pos" represents the significant metabolite features obtained from ESI+ (801 features) and a sheet "Supplementary Table 1 Neg" represents the significant metabolite features obtained from ESI- (444 features) modes. Significant metabolite features labelled in red color with bold letter showed obvious alteration patterns in cluster analysis.

Supplementary Table 2. Mean \pm SD ( $n=3 X 3$ /group) of metabolite features based on their alteration pattern with level changes $>1.5$ folds were analyzed by Mann-Whitney $\mathrm{U}$ test to select significant differences between control and treatment. A sheet "Supplementary Table 2_Pos" represents 40 metabolite features and a sheet "Supplementary Table 2_Neg" represents 56 metabolite features. 
Supplementary Table 3. Summary of the metabolite annotation and identification of the

significant metabolite features

\section{References}

Abubakirov, N.K. (1980). New Phytoecdysones. IN Ananchenko, S.N. (Ed.) Frontiers of Bioorganic Chemistry and Molecular Biology. Pergamon.

Adler, J.H. \& Grebenok, R.J. (1999). Occurrence, Biosynthesis, and Putative Role of Ecdysteroids in Plants. Critical Reviews in Biochemistry and Molecular Biology, 34: 253-264. DOI 10.1080/10409239991209282

Allwood, J.W. \& Goodacre, R. (2010). An introduction to liquid chromatography-mass spectrometry instrumentation applied in plant metabolomic analyses. Phytochemical Analysis, 21: 33-47. DOI 10.1002/pca.1187

Allwood, J.W., Woznicki, T.L., Xu, Y., Foito, A., Aaby, K., Sungurtas, J., Freitag, S., Goodacre, R., Stewart, D., Remberg, S.F., Heide, O.M. \& Sønsteby, A. (2019). Application of HPLC-PDA-MS metabolite profiling to investigate the effect of growth temperature and day length on blackcurrant fruit. Metabolomics, 15: 12. DOI 10.1007/s11306-018-1462-5

Ashihara, H., Stasolla, C., Fujimura, T. \& Crozier, A. (2018). Purine salvage in plants. Phytochemistry, 147: 89-124. DOI https://doi.org/10.1016/j.phytochem.2017.12.008

Balmer, D., Flors, V., Glauser, G. \& Mauch-Mani, B. (2013). Metabolomics of cereals under biotic stress: current knowledge and techniques. Frontiers in Plant Science, 4. DOI 10.3389/fpls.2013.00082

Baltayev, U.A. (1998). Ecdysteroside, a phytoecdysteroid from Silene tatarica. Phytochemistry, 47: 1233-1235. DOI https://doi.org/10.1016/S0031-9422(97)00715-2

Biais, B., Allwood, J.W., Deborde, C., Xu, Y., Maucourt, M., Beauvoit, B., Dunn, W.B., Jacob, D., Goodacre, R., Rolin, D. \& Moing, A. (2009). 1H NMR, GC-EI-TOFMS, and Data Set Correlation for Fruit Metabolomics: Application to Spatial Metabolite Analysis in Melon. Analytical Chemistry, 81: 2884-2894. DOI 10.1021/ac9001996

Broadhurst, D., Goodacre, R., Reinke, S.N., Kuligowski, J., Wilson, I.D., Lewis, M.R. \& Dunn, W.B. (2018). Guidelines and considerations for the use of system suitability and quality control samples in mass spectrometry assays applied in untargeted clinical metabolomic studies. Metabolomics : Official journal of the Metabolomic Society, 14: 72-72. DOI 10.1007/s11306-018-1367-3

Brychkova, G., Fluhr, R. \& Sagi, M. (2008). Formation of xanthine and the use of purine metabolites as a nitrogen source in Arabidopsis plants. Plant Signaling \& Behavior, 3: 999-1001. DOI 10.4161/psb.6304

Carrizo, D., Chevallier, O.P., Woodside, J.V., Brennan, S.F., Cantwell, M.M., Cuskelly, G. \& Elliott, C.T. (2017). Untargeted metabolomic analysis of human serum samples associated with exposure levels of Persistent organic pollutants indicate important perturbations in Sphingolipids and Glycerophospholipids levels. Chemosphere, 168: 731-738. DOI https://doi.org/10.1016/j.chemosphere.2016.11.001

Chaubey, M.K. (2018). Role of Phytoecdysteroids in Insect Pest Management: A Review. Journal of Agronomy, 17: 1-10.

Clifford, Michael n., Jaganath, I.B., Ludwig, I.A. \& Crozier, A. (2017). Chlorogenic acids and the acyl-quinic acids: discovery, biosynthesis, bioavailability and bioactivity. Natural Product Reports, 34: 1391-1421. DOI 10.1039/C7NP00030H 
Csábi, J., Martins, A., Sinka, I., Csorba, A., Molnár, J., Zupkó, I., Tóth, G., Tillekeratne, L.M.V. \& Hunyadi, A. (2016). Synthesis and in vitro evaluation of the antitumor potential and chemo-sensitizing activity of fluorinated ecdysteroid derivatives. MedChemComm, 7: 2282-2289. DOI 10.1039/C6MD00431H

Dinan, L. (2001). Phytoecdysteroids: biological aspects. Phytochemistry, 57: 325-339. DOI https://doi.org/10.1016/S0031-9422(01)00078-4

Dreyer, D.L. \& Jones, K.C. (1981). Feeding deterrency of flavonoids and related phenolics towards Schizaphis graminum and Myzus persicae: Aphid feeding deterrents in wheat. Phytochemistry, 20: 2489-2493. DOI https://doi.org/10.1016/00319422(81)83078-6

Du, S.S., Zhang, H.M., Bai, C.Q., Wang, C.F., Liu, Q.Z., Liu, Z.L., Wang, Y.Y. \& Deng, Z.W. (2011). Nematocidal Flavone-C-Glycosides against the Root-Knot Nematode (Meloidogyne incognita) from Arisaema erubescens Tubers. Molecules, 16: 5079.

Dunn, W.B., Broadhurst, D., Begley, P., Zelena, E., Francis-Mcintyre, S., Anderson, N., Brown, M., Knowles, J.D., Halsall, A., Haselden, J.N., Nicholls, A.W., Wilson, I.D., Kell, D.B. \& Goodacre, R. (2011). Procedures for large-scale metabolic profiling of serum and plasma using gas chromatography and liquid chromatography coupled to mass spectrometry. Nat Protocols, 6: 1060-1083. DOI http://www.nature.com/nprot/journal/v6/n7/abs/nprot.2011.335.html\#supplementaryinformation

Eisen, M.B., Spellman, P.T., Brown, P.O. \& Botstein, D. (1998). Cluster analysis and display of genome-wide expression patterns. Proceedings of the National Academy of Sciences, 95: 14863-14868.

Falcone Ferreyra, M.L., Rius, S. \& Casati, P. (2012). Flavonoids: Biosynthesis, Biological functions and Biotechnological applications. Frontiers in Plant Science, 3. DOI 10.3389/fpls.2012.00222

Felton, G.W., Donato, K., Del Vecchio, R.J. \& Duffey, S.S. (1989). Activation of plant foliar oxidases by insect feeding reduces nutritive quality of foliage for noctuid herbivores. Journal of Chemical Ecology, 15: 2667-2694. DOI 10.1007/BF01014725

Felton, G.W. \& Duffey, S.S. (1990). Inactivation of baculovirus by quinones formed in insect-damaged plant tissues. Journal of Chemical Ecology, 16: 1221-1236. DOI 10.1007/bf01021021

Fujita, D., Kohli, A. \& Horgan, F.G. (2013). Rice Resistance to Planthoppers and Leafhoppers. Critical Reviews in Plant Sciences, 32: 162-191. DOI 10.1080/07352689.2012.735986

Gattuso, G., Barreca, D., Gargiulli, C., Leuzzi, U. \& Caristi, C. (2007). Flavonoid composition of Citrus juices. Molecules (Basel, Switzerland), 12: 1641-1673. DOI 10.3390/12081641

Gattuso, G., Caristi, C., Gargiulli, C., Bellocco, E., Toscano, G. \& Leuzzi, U. (2006). Flavonoid Glycosides in Bergamot Juice (Citrus bergamia Risso). Journal of Agricultural and Food Chemistry, 54: 3929-3935. DOI 10.1021/jf060348z

Grayer, R.J., Harborne, J.B., Kimmins, F.M., Stevenson, P.C. \& Wijayagunasekera, H.N.P. (1994). Phenolics in rice phloem sap as sucking deterrents to the brown planhopper, Nilapavata lugens. 381 ed.: International Society for Horticultural Science (ISHS), Leuven, Belgium.

Hamilton, M.L., Kuate, S.P., Brazier-Hicks, M., Caulfield, J.C., Rose, R., Edwards, R., Torto, B., Pickett, J.A. \& Hooper, A.M. (2012). Elucidation of the biosynthesis of the di-Cglycosylflavone isoschaftoside, an allelopathic component from Desmodium spp. that inhibits Striga spp. development. Phytochemistry, 84: 169-176. DOI http://dx.doi.org/10.1016/j.phytochem.2012.08.005 
Hao, P.-Y., Feng, Y.-L., Zhou, Y.-S., Song, X.-M., Li, H.-L., Ma, Y., Ye, C.-L. \& Yu, X.-P. (2018). Schaftoside Interacts With NICDK1 Protein: A Mechanism of Rice Resistance to Brown Planthopper, Nilaparvata lugens. Frontiers in Plant Science, 9. DOI 10.3389/fpls.2018.00710

Harborne, J.B. \& Williams, C.A. (2000). Advances in flavonoid research since 1992. Phytochemistry, 55: 481-504. DOI http://dx.doi.org/10.1016/S0031-9422(00)00235-1

Harrison, H., Mitchell, T.R., Peterson, J.K., Wechter, W.P., Majetich, G.F. \& Snook, M.E. (2008). Contents of Caffeoylquinic Acid Compounds in the Storage Roots of Sixteen Sweetpotato Genotypes and Their Potential Biological Activity. Journal of the American Society for Horticultural Science, 133.

Hauck, O.K., Scharnberg, J., Escobar, N.M., Wanner, G., Giavalisco, P. \& Witte, C.-P. (2014). Uric Acid Accumulation in an Arabidopsis Urate Oxidase Mutant Impairs Seedling Establishment by Blocking Peroxisome Maintenance. The Plant Cell, 26: 3090. DOI 10.1105/tpc.114.124008

Heinrichs, E.A. (1979). Brown Planthopper: Threat to Rice Production in Asia. IN A., H.E. (Ed.) Chemical control of the brown planthopper. Philippines: International Rice Research Institute.

Heong, K.L.a.H., B. (2009). Planthopper: New treats to the sustainability of intensive rice production systems in Asia. Los Banos (Philippines): International Rice Research Institute.

Hu, J., Xiao, C. \& He, Y. (2016). Recent progress on the genetics and molecular breeding of brown planthopper resistance in rice. Rice (New York, NY), 9: 30-30. DOI 10.1186/s12284-016-0099-0

Jairin, J., Teangdeerith, S., Leelagud, P., Kothcharerk, J., Sansen, K., Yi, M., Vanavichit, A. \& Toojinda, T. (2009). Development of rice introgression lines with brown planthopper resistance and KDML105 grain quality characteristics through markerassisted selection. Field Crops Research, 110: 263-271. DOI http://dx.doi.org/10.1016/j.fcr.2008.09.009

Kamolsukyeunyong, W., Ruengphayak, S., Chumwong, P., Kusumawati, L., Chaichoompu, E., Jamboonsri, W., Saensuk, C., Phoonsiri, K., Toojinda, T. \& Vanavichit, A. (2019). Identification of spontaneous mutation for broad-spectrum brown planthopper resistance in a large, long-term fast neutron mutagenized rice population. Rice, 12: 126. DOI 10.1186/s12284-019-0274-1

Kamolsukyunyong, W., Sukhaket, W., Ruanjaichon, V., Toojinda, T. \& Vanavichit, A. (2013). Single-feature polymorphism mapping of isogenic rice lines identifies the influence of terpene synthase on brown planthopper feeding preferences. Rice, 6: 18.

Kang, K., Yue, L., Xia, X., Liu, K. \& Zhang, W. (2019). Comparative metabolomics analysis of different resistant rice varieties in response to the brown planthopper Nilaparvata lugens Hemiptera: Delphacidae. Metabolomics, 15: 62. DOI 10.1007/s11306-0191523-4

Kessler, A. \& Baldwin, I.T. (2002). Plant responses to insect herbivory: The emerging molecular analysis. Annual Review of Plant Biology, 53. DOI 10.1146/annurev.arplant.53.100301.135207

Kuijpers, T.F.M., Narváez-Cuenca, C.-E., Vincken, J.-P., Verloop, A.J.W., Van Berkel, W.J.H. \& Gruppen, H. (2012). Inhibition of Enzymatic Browning of Chlorogenic Acid by Sulfur-Containing Compounds. Journal of Agricultural and Food Chemistry, 60: 3507-3514. DOI 10.1021/jf205290w

Kundu, A. \& Vadassery, J. (2019). Chlorogenic acid-mediated chemical defence of plants against insect herbivores. Plant Biology, 21: 185-189. DOI 10.1111/plb.12947 
Kusumawati, L., Chumwong, P., Jamboonsri, W., Wanchana, S., Siangliw, J.L., Siangliw, M., Khanthong, S., Vanavichit, A., Kamolsukyeunyong, W. \& Toojinda, T. (2018). Candidate genes and molecular markers associated with brown planthopper (Nilaparvata lugens Stål) resistance in rice cultivar Rathu Heenati. Mol Breeding, 38: 88. DOI 10.1007/s11032-018-0847-5

Li, X., Grand, L., Pouleriguen, T., Queneau, Y., Da Silva, P., Rahbé, Y., Poëssel, J.-L. \& Moebs-Sanchez, S. (2016). Synthesis of new dicinnamoyl 4-deoxy quinic acid and methyl ester derivatives and evaluation of the toxicity against the pea aphid Acyrthosiphon pisum. Organic \& Biomolecular Chemistry, 14: 2487-2497. DOI 10.1039/C5OB02483H

Li, Z., Xue, Y., Zhou, H., Li, Y., Usman, B., Jiao, X., Wang, X., Liu, F., Qin, B., Li, R. \& Qiu, Y. (2019). High-resolution mapping and breeding application of a novel brown planthopper resistance gene derived from wild rice (Oryza. rufipogon Griff). Rice, 12: 41. DOI 10.1186/s12284-019-0289-7

Liu, C., Du, B., Hao, F., Lei, H., Wan, Q., He, G., Wang, Y. \& Tang, H. (2017). Dynamic metabolic responses of brown planthoppers towards susceptible and resistant rice plants. Plant biotechnology journal, 15: 1346-1357. DOI 10.1111/pbi.12721

Liu, C., Hao, F., Hu, J., Zhang, W., Wan, L., Zhu, L., Tang, H. \& He, G. (2010). Revealing Different Systems Responses to Brown Planthopper Infestation for Pest Susceptible and Resistant Rice Plants with the Combined Metabonomic and Gene-Expression Analysis. Journal of Proteome Research, 9: 6774-6785. DOI 10.1021/pr100970q

Liu, Y., Wu, H., Chen, H., Liu, Y., He, J., Kang, H., Sun, Z., Pan, G., Wang, Q., Hu, J., Zhou, F., Zhou, K., Zheng, X., Ren, Y., Chen, L., Wang, Y., Zhao, Z., Lin, Q., Wu, F., Zhang, X., Guo, X., Cheng, X., Jiang, L., Wu, C., Wang, H. \& Wan, J. (2015). A gene cluster encoding lectin receptor kinases confers broad-spectrum and durable insect resistance in rice. Nat Biotech, 33: 301-305. DOI 10.1038/nbt.3069

http://www.nature.com/nbt/journal/v33/n3/abs/nbt.3069.html\#supplementary-information Martinez, V., Mestre, T.C., Rubio, F., Girones-Vilaplana, A., Moreno, D.A., Mittler, R. \& Rivero, R.M. (2016). Accumulation of Flavonols over Hydroxycinnamic Acids Favors Oxidative Damage Protection under Abiotic Stress. Frontiers in plant science, 7: 838-838. DOI 10.3389/fpls.2016.00838

Matsui, T., Ebuchi, S., Fujise, T., Abesundara, K.J.M., Doi, S., Yamada, H. \& Matsumoto, K. (2004). Strong Antihyperglycemic Effects of Water-Soluble Fraction of Brazilian Propolis and Its Bioactive Constituent, 3,4,5-Tri-<i $>\mathrm{O}</ \mathrm{i}>$-caffeoylquinic Acid. Biological and Pharmaceutical Bulletin, 27: 1797-1803. DOI 10.1248/bpb.27.1797

Mierziak, J., Kostyn, K. \& Kulma, A. (2014). Flavonoids as Important Molecules of Plant Interactions with the Environment. Molecules, 19. DOI 10.3390/molecules 191016240

Moglia, A., Lanteri, S., Comino, C., Acquadro, A., De Vos, R. \& Beekwilder, J. (2008). Stress-Induced Biosynthesis of Dicaffeoylquinic Acids in Globe Artichoke. Journal of Agricultural and Food Chemistry, 56: 8641-8649. DOI 10.1021/jf801653w Moglia, A., Lanteri, S., Comino, C., Hill, L., Knevitt, D., Cagliero, C., Rubiolo, P., Bornemann, S. \& Martin, C. (2014). Dual catalytic activity of hydroxycinnamoylcoenzyme A quinate transferase from tomato allows it to moonlight in the synthesis of both mono- and dicaffeoylquinic acids. Plant physiology, 166: 1777-1787. DOI 10.1104/pp.114.251371

Nakabayashi, R. \& Saito, K. (2015). Integrated metabolomics for abiotic stress responses in plants. Current Opinion in Plant Biology, 24: 10-16. DOI https://doi.org/10.1016/j.pbi.2015.01.003 
O'kane, A.A., Chevallier, O.P., Graham, S.F., Elliott, C.T. \& Mooney, M.H. (2013). Metabolomic Profiling of In Vivo Plasma Responses to Dioxin-Associated Dietary Contaminant Exposure in Rats: Implications for Identification of Sources of Animal and Human Exposure. Environmental Science \& Technology, 47: 5409-5418. DOI $10.1021 / \mathrm{es} 305345 \mathrm{u}$

Pastor, V., Balmer, A., Gamir, J., Flors, V. \& Mauch-Mani, B. (2014). Preparing to fight back: generation and storage of priming compounds. Frontiers in Plant Science, 5. DOI 10.3389/fpls.2014.00295

Pierpoint, W.S. (1969). O-Quinones formed in plant extracts. Their reaction with bovine serum albumin. Biochemical Journal, 112: 619. DOI 10.1042/bj1120619

Pitija, K., Kamolsukyumyong, W., Vanavichit, A., Sookwong, P. \& Mahatheeranont, S. (2014). Monoterpenoid Allelochemicals in Resistance Rice Varieties against Brown Planthoppers, Nilaparvata Lugens (Stål). Journal of Advanced Agricultural Technologies, 1: 7. DOI 10.12720/joaat.1.2.82-88

Refaat, J., Desoukey, S.Y., Ramadan, M.A. \& Kamel, M.S. (2015). RHOIFOLIN: A REVIEW OF SOURCES AND BIOLOGICAL ACTIVITIES. International Journal of Pharmacognosy, 2.

Ren, J., Gao, F., Wu, X., Lu, X., Zeng, L., Lv, J., Su, X., Luo, H. \& Ren, G. (2016). Bph32, a novel gene encoding an unknown SCR domain-containing protein, confers resistance against the brown planthopper in rice. Scientific reports, 6: 37645-37645. DOI 10.1038/srep37645

Santos, I.C.D., Almeida, A.-a.F.D., Pirovani, C.P., Costa, M.G.C., Silva, M.F.D.G.F.D., Bellete, B.S., Freschi, L., Soares Filho, W., Coelho Filho, M.A. \& Gesteira, A.D.S. (2017). Differential accumulation of flavonoids and phytohormones resulting from the canopy/rootstock interaction of citrus plants subjected to dehydration/rehydration. Plant Physiology and Biochemistry, 119: 147-158. DOI https://doi.org/10.1016/j.plaphy.2017.08.019

Schmelz, E.A., Grebenok, R.J., Ohnmeiss, T.E. \& Bowers, W.S. (2002). Interactions between Spinacia oleracea and Bradysia impatiens: A role for phytoecdysteroids. Archives of Insect Biochemistry and Physiology, 51: 204-221. DOI 10.1002/arch.10062

Slámová, K., Kapešová, J. \& Valentová, K. (2018). "Sweet Flavonoids": GlycosidaseCatalyzed Modifications. Int J Mol Sci, 19: 2126. DOI 10.3390/ijms19072126

Smilde, A.K., Westerhuis, J.A. \& De Jong, S. (2003). A framework for sequential multiblock component methods. Journal of Chemometrics, 17: 323-337. DOI 10.1002/cem.811

Stevenson, P.C., Kimmins, F.M., Grayer, R.J. \& Raveendranath, S. (1996). Schaftosides from rice phloem as feeding inhibitors and resistance factors to brown planthoppers, Nilaparvata lugens. IN Städler, E., Rowell-Rahier, M. \& Bauer, R. (Eds.) Proceedings of the 9th International Symposium on Insect-Plant Relationships. Springer Netherlands.

Sumner, L.W., Amberg, A., Barrett, D., Beale, M.H., Beger, R., Daykin, C.A., Fan, T.W.M., Fiehn, O., Goodacre, R., Griffin, J.L., Hankemeier, T., Hardy, N., Harnly, J., Higashi, R., Kopka, J., Lane, A.N., Lindon, J.C., Marriott, P., Nicholls, A.W., Reily, M.D., Thaden, J.J. \& Viant, M.R. (2007). Proposed minimum reporting standards for chemical analysis Chemical Analysis Working Group (CAWG) Metabolomics Standards Initiative (MSI). Metabolomics : Official journal of the Metabolomic Society, 3: 211-221. DOI 10.1007/s11306-007-0082-2

Tenango, M.P., Hernández, M.S. \& Hernández, E.A. (2017). Flavonoids in Agriculture, Flavonoids - From Biosynthesis to Human Health. IN Justino, G.C. (Ed.): IntechOpen. 
Toojinda, T., Tragoonrung, S., Vanavichit, A., Siangliw, J.L., Pa-In, N., Jantaboon, J., Siangliw, M. \& Fukai, S. (2005). Molecular Breeding for Rainfed Lowland Rice in the Mekong Region. Plant Production Science, 8: 330-333.

Uawisetwathana, U., Graham, S., Kamolsukyunyong, W., Sukhaket, W., Klanchui, A., Toojinda, T., Vanavichit, A., Karoonuthaisiri, N. \& Elliott, C. (2015). Quantitative 1H NMR metabolome profiling of Thai Jasmine rice (Oryza sativa) reveals primary metabolic response during brown planthopper infestation. Metabolomics, 11: 16401655. DOI 10.1007/s11306-015-0817-4

War, A.R., Paulraj, M.G., Ahmad, T., Buhroo, A.A., Hussain, B., Ignacimuthu, S. \& Sharma, H.C. (2012). Mechanisms of plant defense against insect herbivores. Plant Signaling \& Behavior, 7: 1306-1320. DOI 10.4161/psb.21663

Wishart, D.S., Jewison, T., Guo, A.C., Wilson, M., Knox, C., Liu, Y., Djoumbou, Y., Mandal, R., Aziat, F., Dong, E., Bouatra, S., Sinelnikov, I., Arndt, D., Xia, J., Liu, P., Yallou, F., Bjorndahl, T., Perez-Pineiro, R., Eisner, R., Allen, F., Neveu, V., Greiner, R. \& Scalbert, A. (2013). HMDB 3.0-The Human Metabolome Database in 2013. Nucleic Acids Research, 41: D801-D807. DOI 10.1093/nar/gks1065

Wittstock, U. \& Gershenzon, J. (2002). Constitutive plant toxins and their role in defense against herbivores and pathogens. Current Opinion in Plant Biology, 5: 300-307. DOI http://dx.doi.org/10.1016/S1369-5266(02)00264-9

Wongpornchai, S., Sriseadka, T. \& Choonvisase, S. (2002). Identification and Quantitation of the Rice Aroma Compound, 2-Acetyl-1-pyrroline, in Bread Flowers (Vallaris glabra Ktze). Journal of Agricultural and Food Chemistry, 51: 457-462. DOI 10.1021/jf025856x

Xu, Y., Correa, E. \& Goodacre, R. (2013). Integrating multiple analytical platforms and chemometrics for comprehensive metabolic profiling: application to meat spoilage detection. Anal Bioanal Chem, 405: 5063-5074. DOI 10.1007/s00216-013-6884-3

$\mathrm{Xu}, \mathrm{Y}$. \& Goodacre, R. (2012). Multiblock principal component analysis: an efficient tool for analyzing metabolomics data which contain two influential factors. Metabolomics, 8: 37-51. DOI 10.1007/s11306-011-0361-9

Zhang, J., Li, Y., Guo, J., Du, B., He, G., Zhang, Y., Chen, R. \& Li, J. (2018). Lipid profiles reveal different responses to brown planthopper infestation for pest susceptible and resistant rice plants. Metabolomics, 14: 120. DOI 10.1007/s11306-018-1422-0 


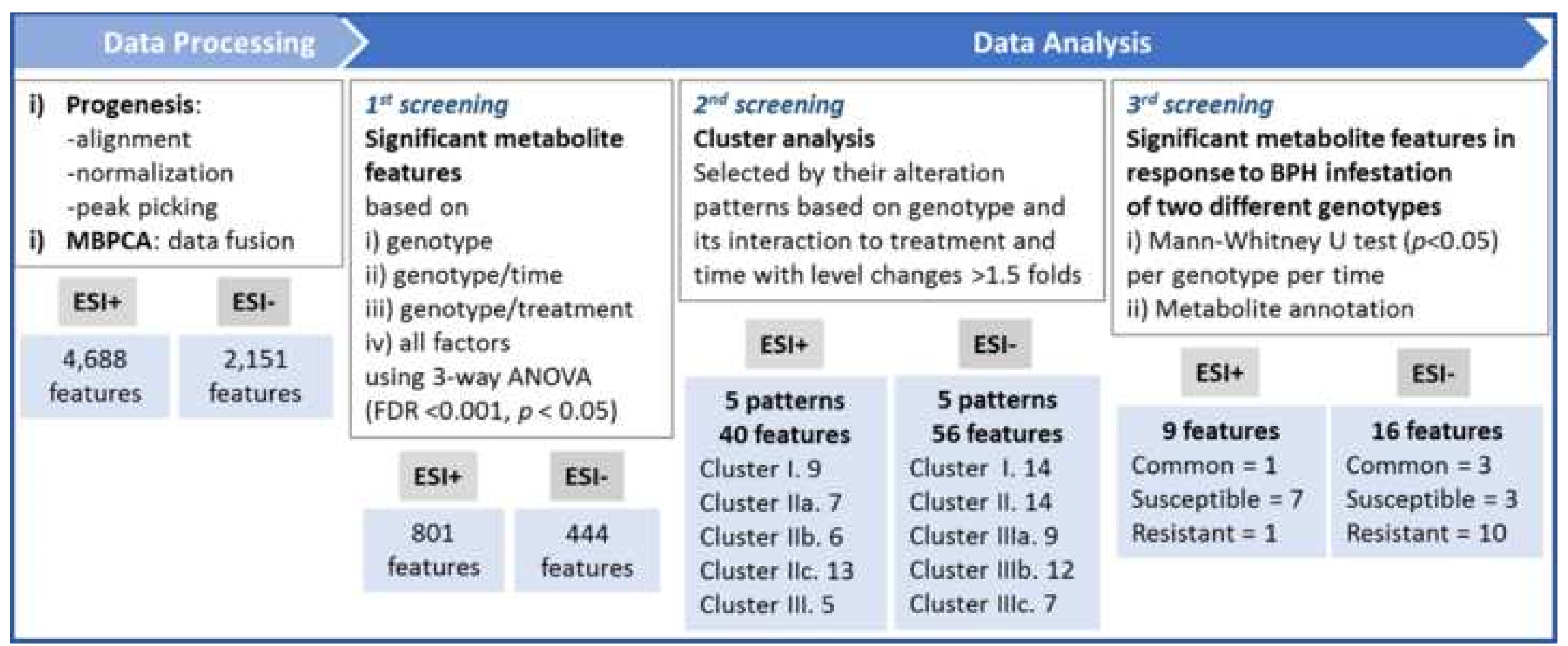




\section{Control}

D1

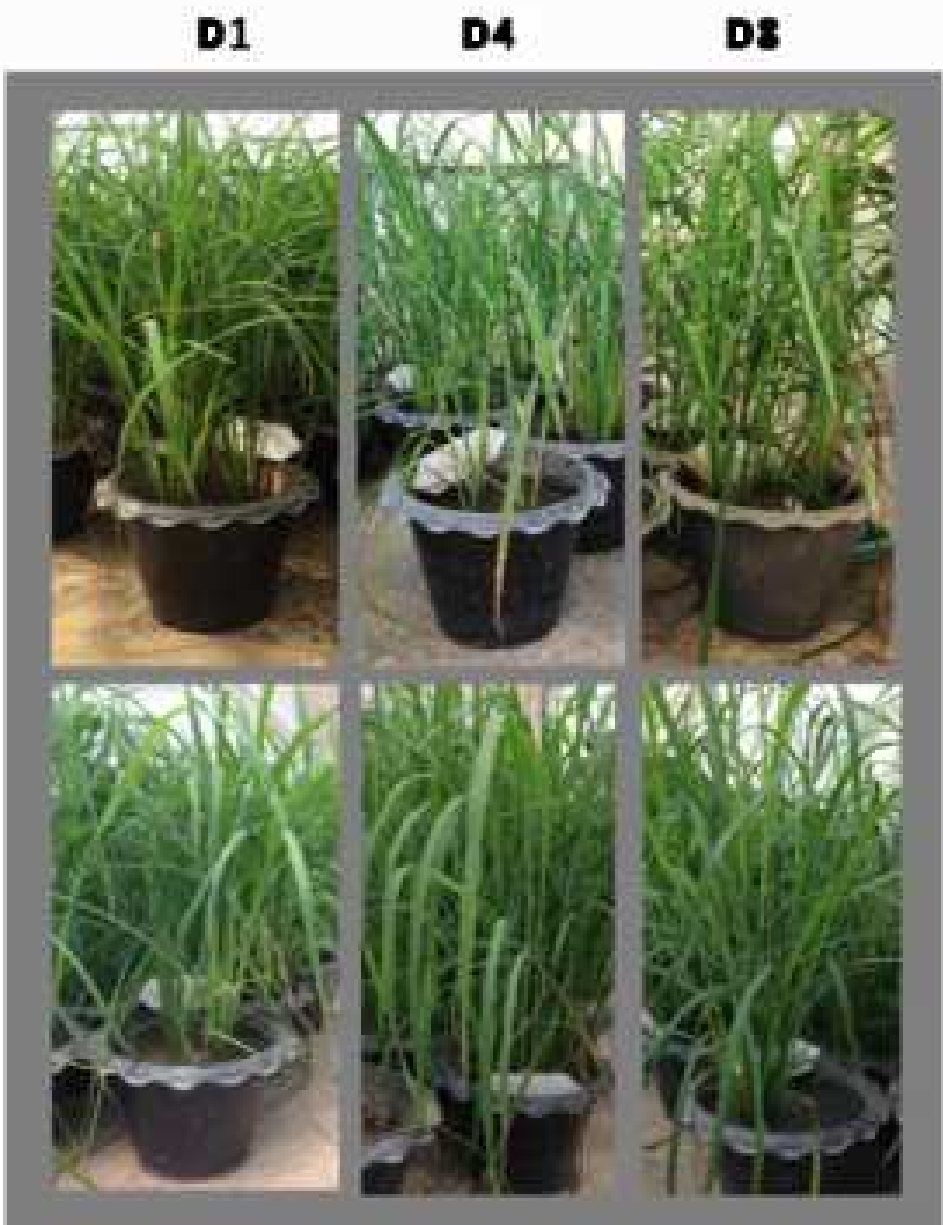

BPH-infested

D1

D4

D8

\section{BPH-susceptible KD variety}

BPH-resistant IL308 variety
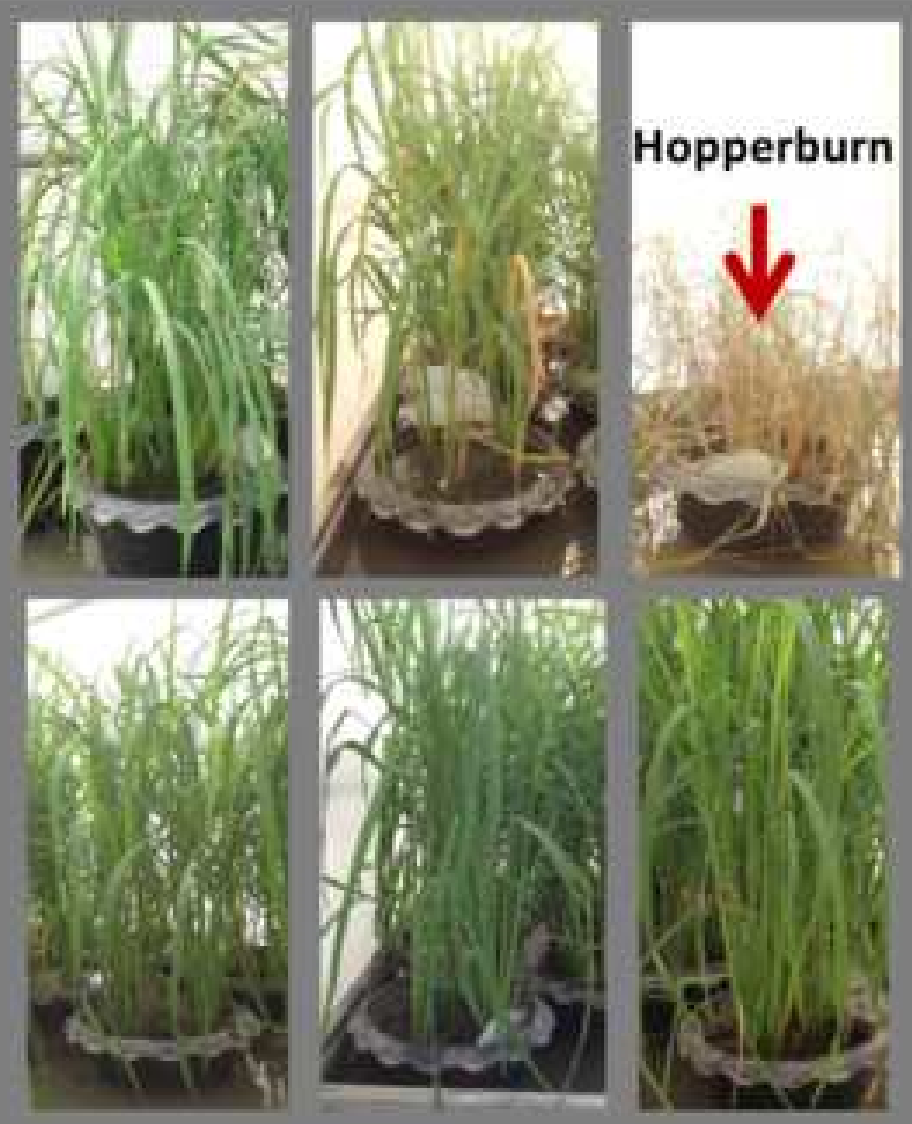
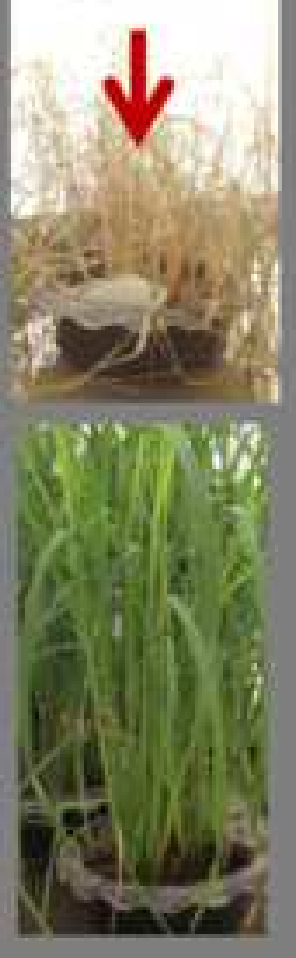

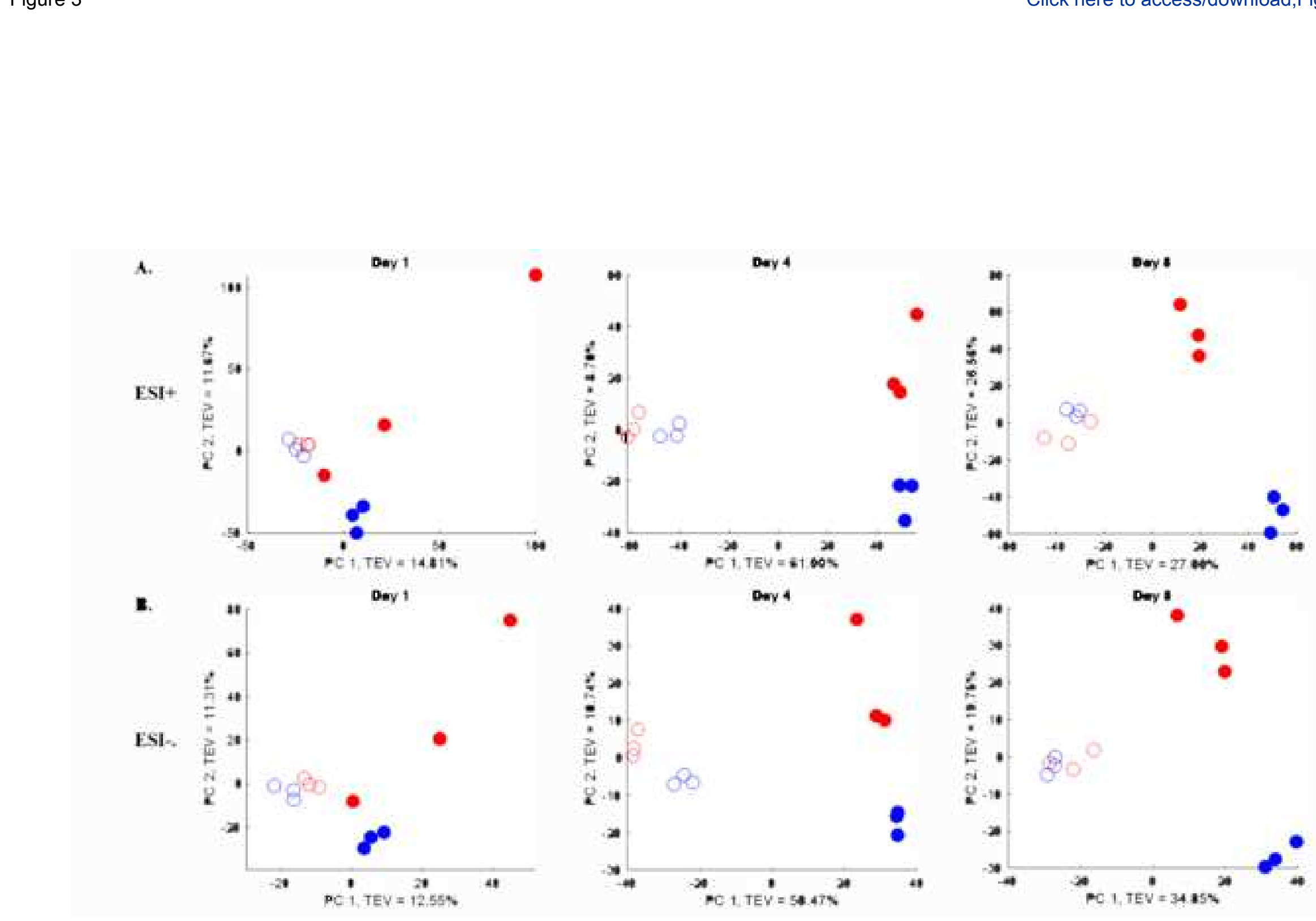

- KD (คDPH)

11308 (-8PH)

- It308 (*aps) 


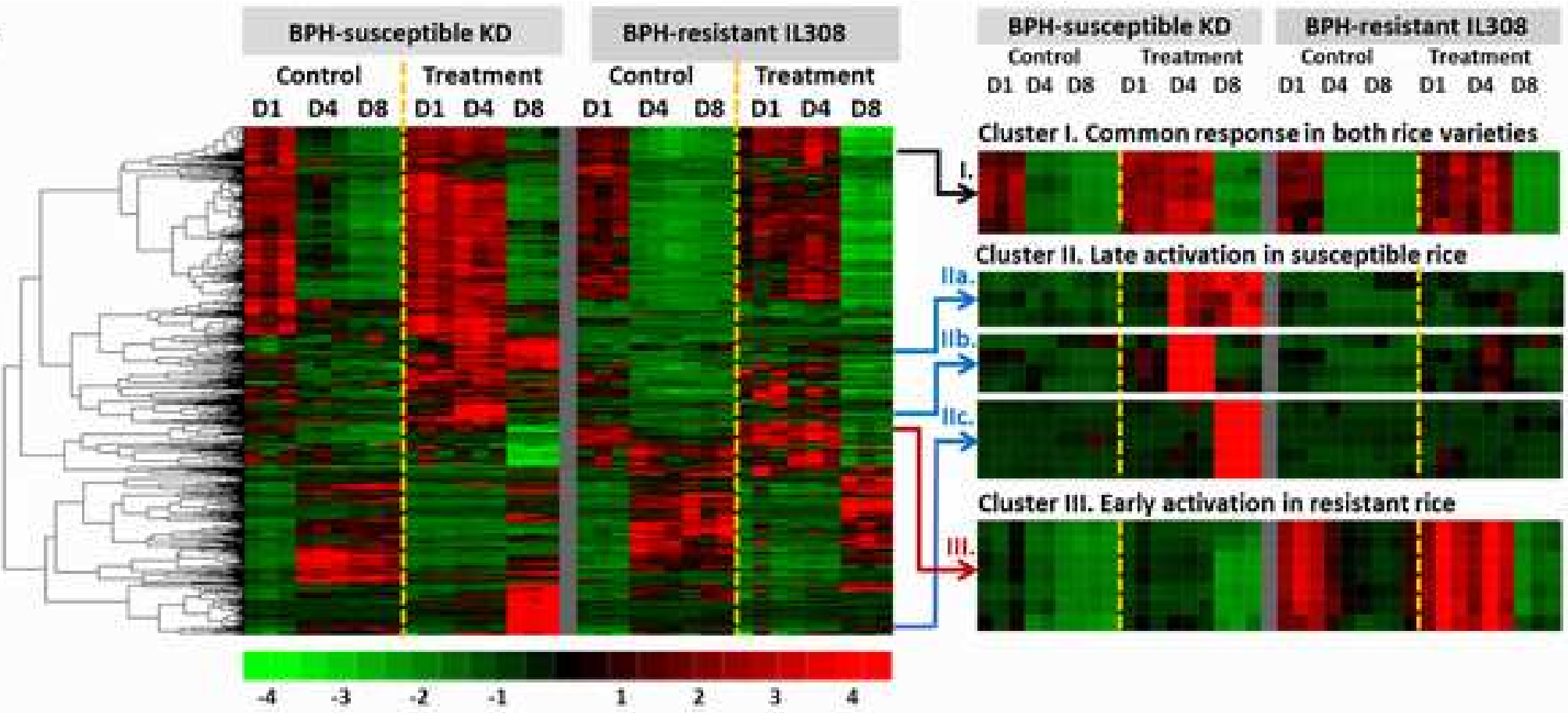

B. ESI-

BPH-susceptible KD

Control Treatment

$\begin{array}{lllllll}D 1 & \text { D4 } & \text { D8 } & \text { D1 } & \text { D4 } & \text { D8 }\end{array}$

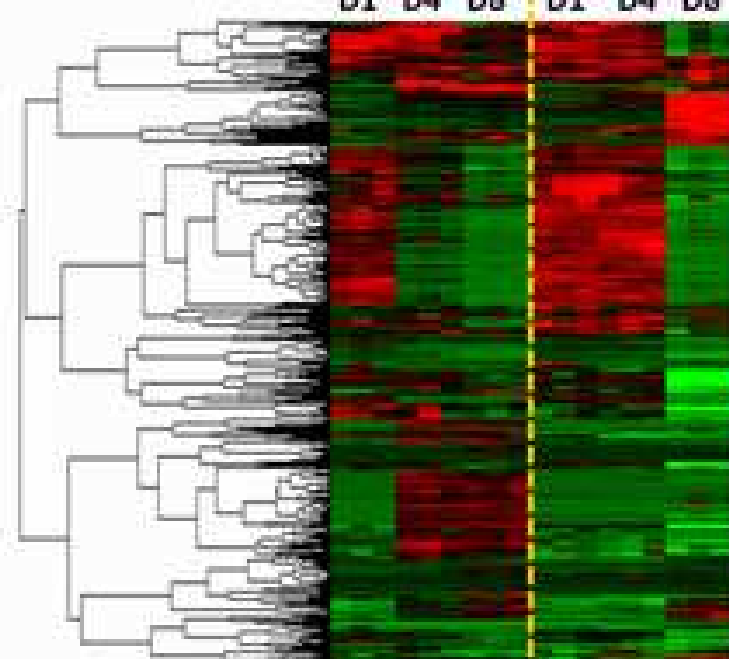

BPH-resistant IL 308

Control Treatment \begin{tabular}{lllllll} 
D1 & D4 & D8 & D1 & D4 & D8 \\
\hline
\end{tabular}

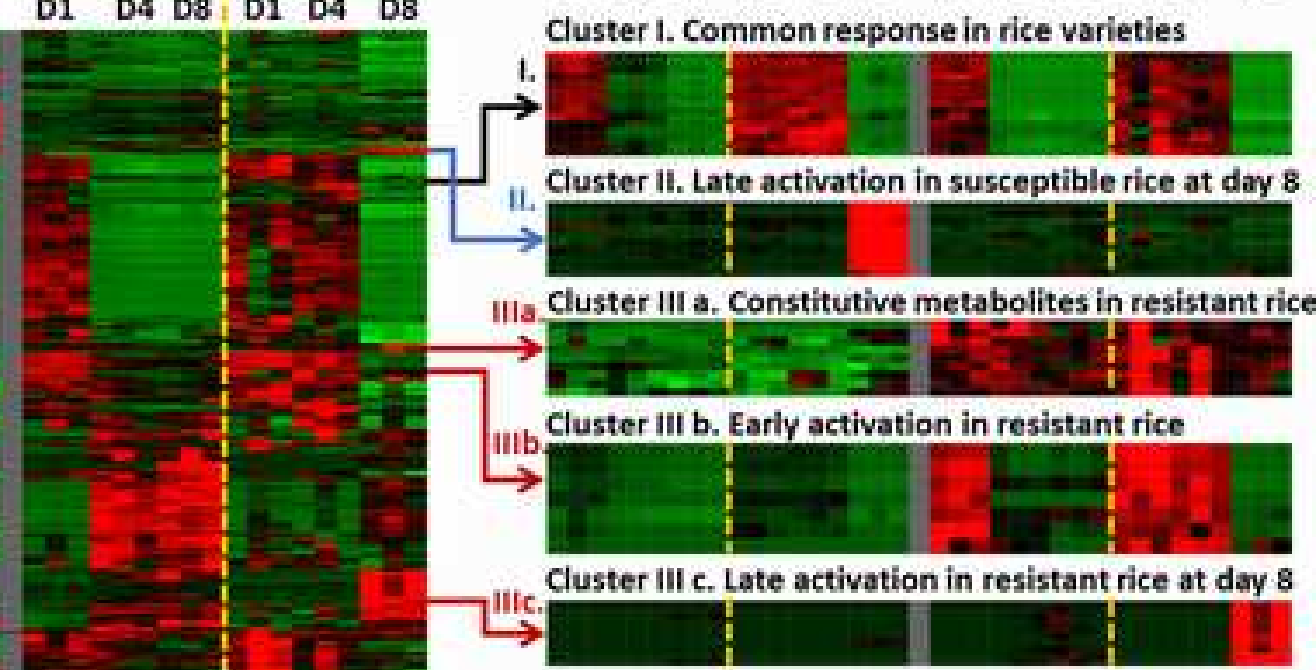
$\rightarrow$

BPH-susceptible KD

Control Treatment

BPH-resistant 11308

Control Treatment $\begin{array}{llllllllllll}\text { D1 } & \text { D4 } & \text { D8 } & \text { D1 } & \text { D4 } & \text { D8 } & \text { D1 } & \text { D4 } & \text { D8 } & \text { D1 } & \text { D4 } & \text { D8 }\end{array}$ 


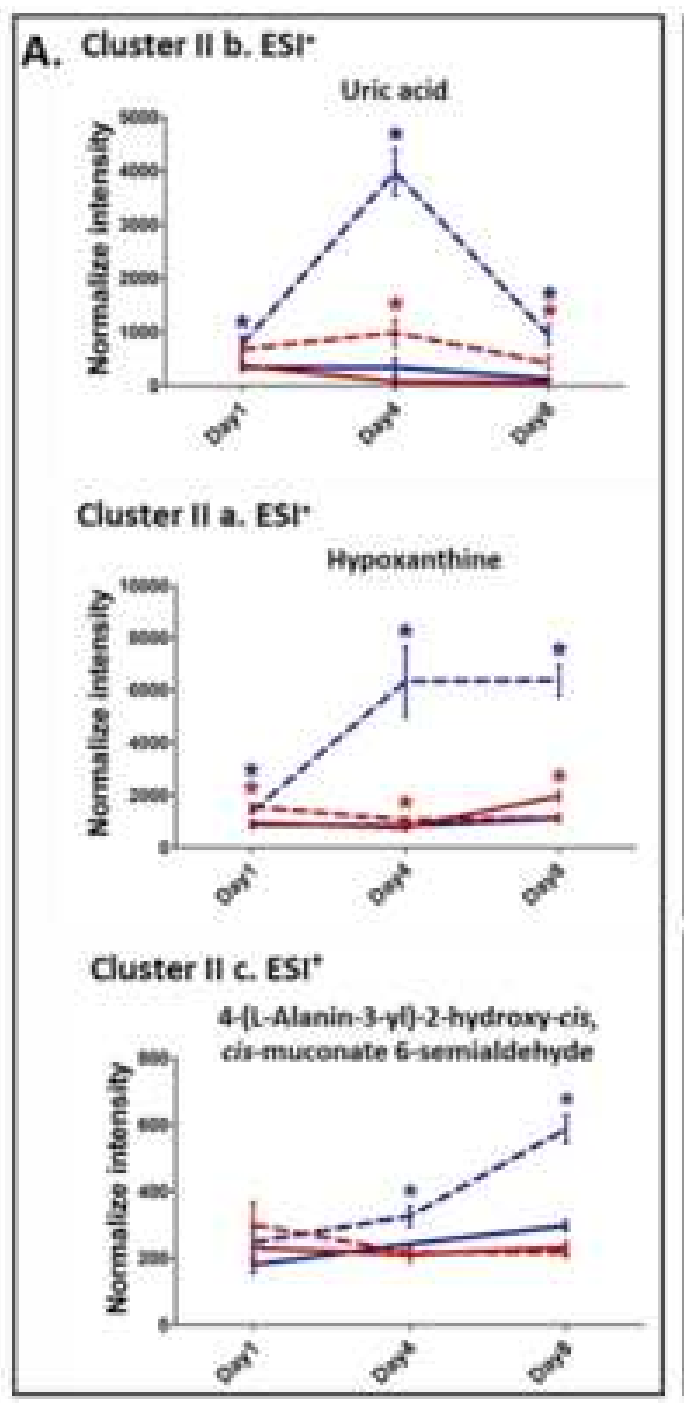

B. Cluster III b. ESI

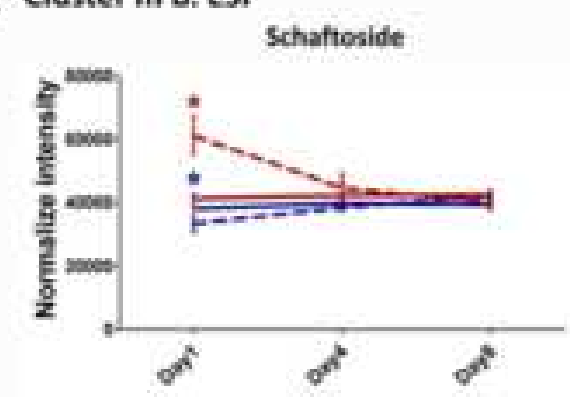

Cluster III b. EST: Apisenin 6-C-a-t-arabinoside-

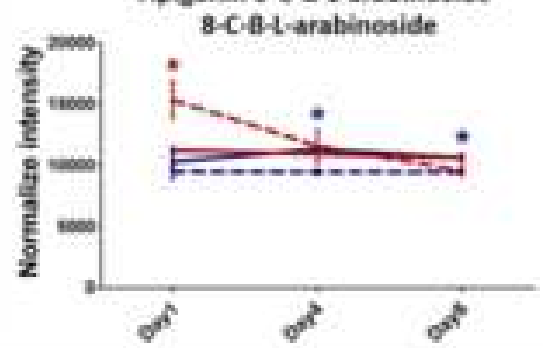

C. Cluster III a. EST

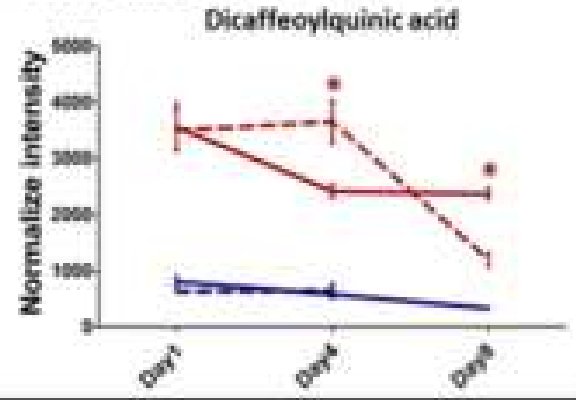

Cluster III b. ESI'

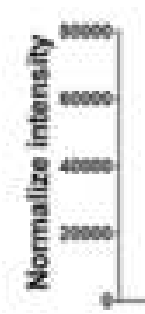

Iso-schaftoside

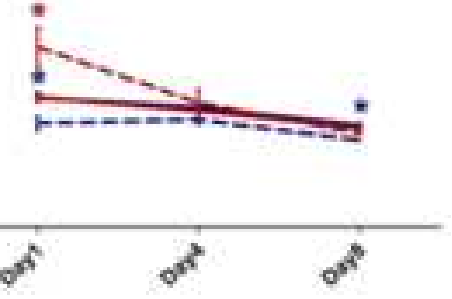

Cluster III b. ESI

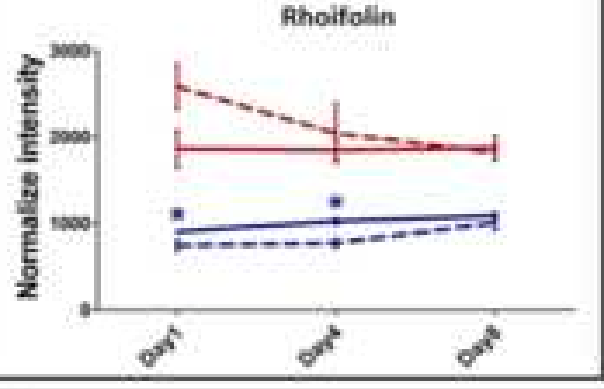

Cluster III a. ESI

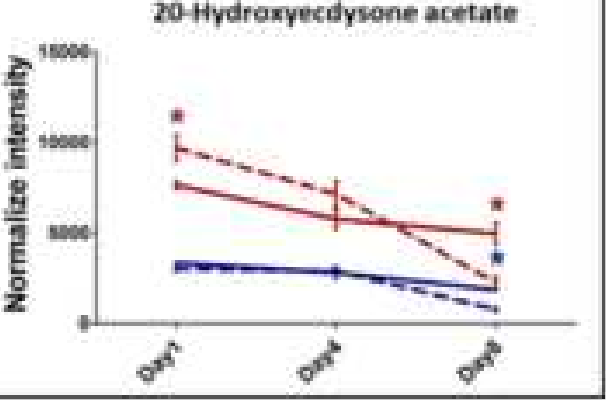

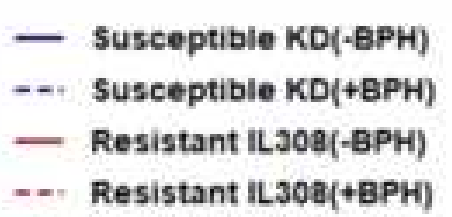




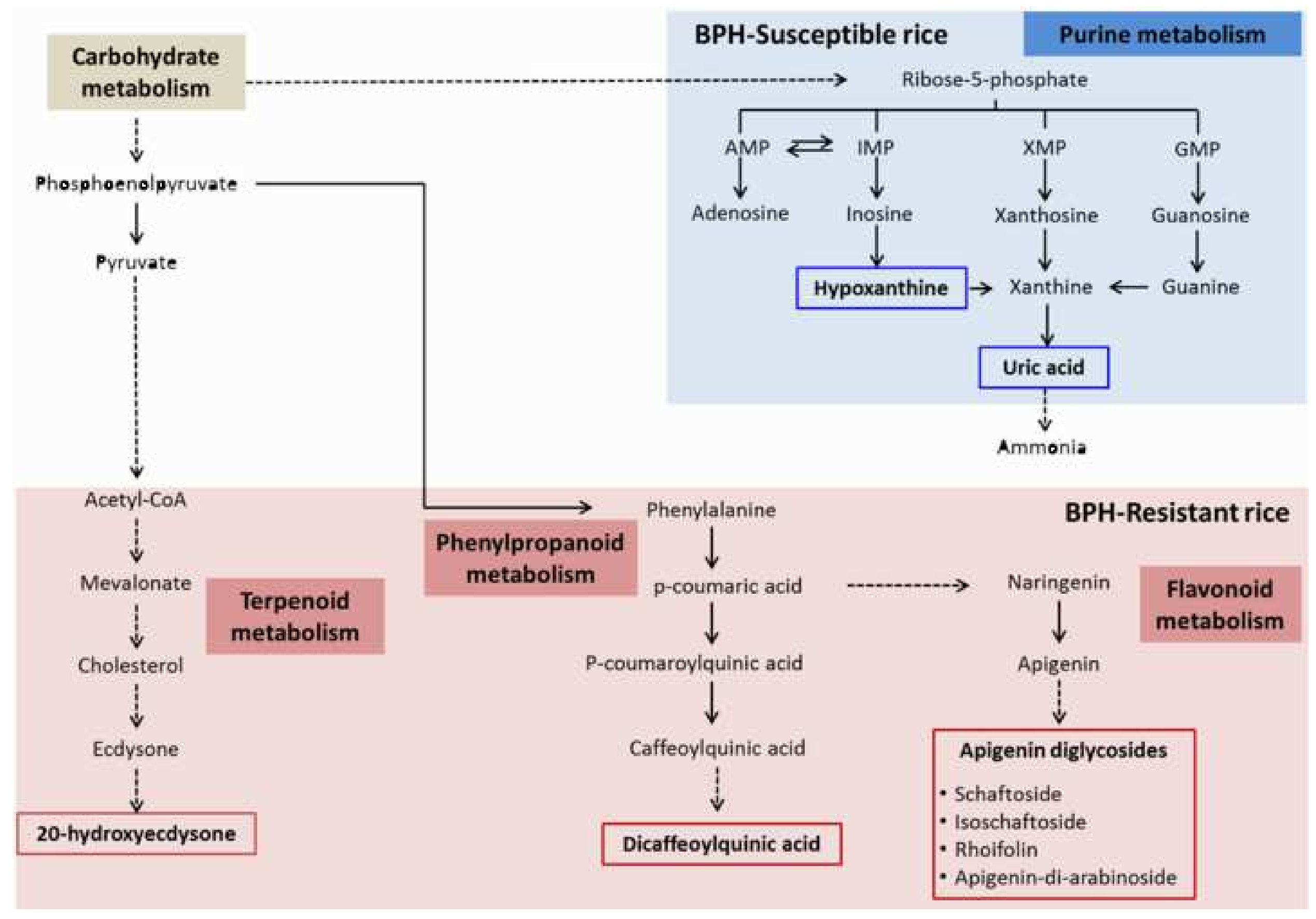

BPH-Susceptible rice

Purine metabolism

metabolism

4

Phosphoenolpyruvate

Pyruvate

Acetyl-CoA

Mevalonate

Terpenoid

Cholesterol

P-coumaroylquinic acid

Caffeoylquinic acid

Dicaffeoylquinic acid 